TRABAJOS DE PREHISTORIA

76, N. ${ }^{\circ}$ 2, julio-diciembre 2019, pp. 199-218, ISSN: 0082-5638

https://doi.org/10.3989/tp.2019.12233

\title{
Análisis bibliométrico de la revista Trabajos de Prehistoria en el contexto de las revistas españolas de Arqueología y Prehistoria recogidas por Scopus e ÍnDICEs CSIC
}

\author{
Bibliometric study of the journal Trabajos de Prehistoria in the context of Spanish \\ journals of Archeology and Prehistory collected by Scopus and ÍnDICEs CSIC
}

\author{
Luis Rodríguez Yunta ${ }^{\mathrm{a}}$, José Ignacio Vidal Liy ${ }^{\mathrm{b}}$ M. ${ }^{\mathrm{a}}$ Isabel Martínez Navarrete
}

\section{RESUMEN}

La publicación en 2018 del volumen 75 de la revista Trabajos de Prehistoria motiva este análisis completo de la misma (1960-2018). El artículo parte de su presencia en bases de datos documentales y se plantea como una aportación al debate sobre la evaluación de las revistas científicas. El estudio bibliométrico aborda la evolución temática, la distribución de la autoría y el impacto a través de citas, a partir de los datos que pueden extraerse de ÍnDICEsCSIC y Scopus. Para situar la revista en el contexto de las publicaciones españolas de Arqueología y Prehistoria, se compara con otras 9 revistas de Arqueología de la península ibérica presentes en Scopus y editadas entre 2010 y 2018 por instituciones públicas: Archivo de Prehistoria Levantina, Archivo Español de Arqueología, Complutum, Lucentum, Munibe Antropologia-Arkeologia, Pyrenae, Saguntum, SPAL y Zephyrus. La clasificación por periodos de los artículos aparecidos en esos 9 años define con claridad la orientación temática de cada revista. Su comparación permite una caracterización general de las pautas de difusión científica de los arqueólogos orientados hacia la Prehistoria y/o hacia la Historia antigua en la última década. En consecuencia, el artículo es también una aportación a la historia de la ciencia.

\section{ABSTRACT}

The publication in 2018 of volume 75 of the journal Trabajos de Prehistoria [TP] provides the occasion for a complete analysis of its contents since its iception in 1960. $\mathrm{TP}$ 's presence in documentary databases makes possible this contribution to the debate on the evaluation of scientific journals. The bibliometric study addresses the thematic evolution, the distribution of authorship and the impact through citations, according to the data that can be extracted from InDICEs-CSIC and Scopus. To place the journal in the context of the Spanish publications of archeology and prehisto$r y$, a comparison is made with other nine Spanish archeology journals present in Scopus and published between 2010 and 2018 by public institutions: Archivo de Prehistoria Levantina, Archivo Español de Arqueología, Complutum, Lucentum, Munibe Antropologia-Arkeologia, Pyrenae, Saguntum, SPAL, and Zephyrus. The classification by periods of the articles that appeared in those 9 years clearly defines the thematic orientation of each journal. Their comparison allows a general characterization of the patterns of scientific publication in the last decade by archeologists oriented towards Prehistory or Ancient History, respectively. Consequently, the article is also a contribution to the history of science.

Palabras clave: Revistas de Prehistoria; Revistas de Arqueología; Península ibérica; Análisis bibliométrico; Evaluación de las revistas; Índices de impacto; Bases de datos documentales; Historia de la ciencia.

Key words: Journals about Prehistory; Journals about Archeology; Iberia; Bibliometric analysis; Journals evaluation; Impact index; Bibliographic databases; History of science.

\footnotetext{
a CSIC, Centro de Ciencias Humanas y Sociales, Unidad de Apoyo a la Edición de Revistas. C/ Albasanz 26-28. 28037 Madrid. España. Correo e.: luis.ryunta@cchs.csic.es https://orcid.org/0000-0002-8424-6205

b CSIC, Centro de Ciencias Humanas y Sociales, Unidad de bases de datos. C/ Albasanz 26-28. 28037 Madrid. España. Correo e.: nacho.vidal@cchs.csic.es https://orcid.org/0000-0001-6169-784X

c CSIC, Centro de Ciencias Humanas y Sociales, Instituto de Historia. C/ Albasanz 26-28. 28037 Madrid. España. Correo e.: isabel.martinez@cchs.csic.es https://orcid.org/0000-0002-3060-6033

Recibido 1-III-2019; aceptado 28-V-2019.
} de uso y distribución "Creative Commons Reconocimiento 4.0 Internacional" (CC BY 4.0) 


\section{INTRODUCCIÓN}

El presente análisis bibliométrico de Trabajos de Prehistoria (en adelante TP) está motivado por la aparición del volumen 75 de esta revista, editada por el Consejo Superior de Investigaciones Científicas (CSIC, Madrid) y una de las publicaciones españolas de arqueología de trayectoria más prolongada e ininterrumpida. Su campo de especialización es la Prehistoria y Protohistoria de la península ibérica y sus relaciones con Europa y el Mediterráneo, de modo que puede considerarse complementaria de Archivo Español de Arqueología (en adelante $A E s p A$ ), también editada por el CSIC desde 1940 y centrada en las culturas desde la Protohistoria a la Alta Edad Media (Mora 2002). El Dr. Martín Almagro Basch, fundador y director de TP (1960-1980), la concibió como serie monográfica con un único estudio por volumen hasta 1968. Pasó a anuario con varios artículos entre 1969 y 1993 y finalmente a semestral a partir de 1994, incorporando el sistema de evaluación por pares (Gilman 2018).

El antecedente de estudio bibliométrico de TP apareció al editarse el volumen 50 y consideraba los trabajos editados entre 1960 y 1992 (Rodríguez Alcalde et al. 1993). Se analizaron temas (periodos y localizaciones geográficas), autoría (investigadores, organismos de filiación y países de procedencia), así como los aspectos formales de la edición. Unos años después se completó con un análisis de las citas recopiladas en las revistas españolas de la disciplina (Rodríguez Alcalde et al. 1996) en el que resultaba la más citada.

$T P$ fue incluida también en los estudios realizados por el Grupo de Investigación de Evaluación de Publicaciones Científicas (EPUC) del CSIC sobre las revistas españolas de Arqueología y Prehistoria (Román Román 2003; Román Román y Alcain Partearroyo 2005). Los resultados de una encuesta a investigadores españoles sirvieron de base para analizar las citas presentes en las revistas mejor valoradas. $T P$ destacaba entre ellas por su mayor número de citas recibidas. Estas investigaciones del grupo EPUC se plasmaron en el portal Revistas Españolas de Ciencias Sociales y Humanidades (RESH), con datos de 2000 a 2008, coincidiendo con el proyecto Índice de impacto de las revistas españolas de ciencias de humanidades (IN-RECH), elaborado por el Grupo de Investigación sobre Evaluación de la Ciencia y de la Comunicación Científica (EC3) de la Universidad de Granada, con datos de 2004 a 2008. Cuando se crearon estos recursos, los índices de citas internacionales no aportaban datos sobre publicaciones españolas de la especialidad. Hay que saludar, por tanto, estas contribuciones como un primer acercamiento a su valoración comparativa. Partiendo de esta aproximación los estudios de citas podían servir para juzgar el trabajo editorial e identificar las principales revistas españolas en las disciplinas de Humanidades y Ciencias Sociales.

En 2009 TP se convierte en la primera publicación española de Arqueología presente en el Journal Citation Reports que establece un ranquin de revistas por el factor de impacto en la plataforma Web of Science (WoS). Igualmente, la presencia en el producto competidor Scopus y en los sistemas de indicadores basados en este producto, le ha dado mayor visibilidad internacional y prestigio, junto a otros títulos españoles seleccionados.

Otra línea de estudios bibliométricos del grupo EC3 maneja los datos de citas recogidas en el portal Google Scholar Metrics, basado en el buscador académico Google Scholar. Esta fuente carece de fiabilidad estadística, pero da una imagen más global de la difusión internacional de las citas. En el proyecto Journal Scholar Metrics (http://www.journal-scholar-metrics.infoec3.es), un ejemplo de estos estudios, TP ocupó la posición 30 sobre 126 revistas internacionales de Arqueología y Prehistoria (primer cuartil) según las citas del periodo 2010-2014.

Desde la época de los primeros estudios bibliométricos sobre $T P$, el panorama general de la edición de revistas científicas se ha transformado notablemente tanto en las formas de distribución y acceso, como en los procesos de gestión y evaluación de las publicaciones (Abadal 2017). Las contribuciones a un dosier sobre revistas científicas de Arqueología editado por M. Bouso et al. (2016) subrayaban cómo la consolidación del sistema de evaluación de la ciencia ha reforzado la profesionalización de la gestión editorial y el rigor en el sistema de evaluadores externos. Las revistas que dependen de instituciones públicas han abordado nuevos retos con mucho voluntarismo. La rigideces administrativas y carencias en recursos materiales y humanos, son frenos para competir con las grandes corporaciones editoriales internacionales. Pero más que el impacto de las nuevas tecnologías y la difusión global, el problema esencial para las revistas sigue siendo "ganar prestigio, para así obtener más propuestas de originales, poder seleccionar los mejores y así reproducir el sistema" (Ruiz Zapatero 2016: 276). En este mismo dosier, Martínez Navarrete y Montero Ruiz (2016) hicieron un balance del proceso de internacionalización de $T P$ en las últimas décadas.

El presente estudio, frente al primero (Rodríguez Alcalde et al. 1993), compara la revista con otras publicaciones españolas de la disciplina con similares características de temática y visibilidad internacional. Su introducción en ese contexto es esencial para su adecuada valoración y es algo usual en cierta medida en los análisis de revistas concretas (Ar- 
mada 2009, 2016). Además, ahora se han podido aprovechar los datos de los índices de citas para las revistas españolas que, al no existir antes, exigían costosos procesos de recopilación (Rodríguez Alcalde et al. 1997).

El primer estudio bibliométrico (Rodríguez Alcalde et al. 1993) tuvo como estímulo la coincidencia del final de los anuarios con la aparición del volumen 50 . Este presente análisis se concibió como una actualización del contenido de la revista 25 años después, incorporable al volumen 75 de 2018. La fuente sería la depuración y aprovechamiento de los datos de la nueva plataforma Información y Documentación de la Ciencia en España (ÍnDICEs CSIC), cuya puesta en marcha se ha producido finalmente en 2018. Este producto es el resultado de la fusión de las bases de datos ICYT (Ciencia y Tecnología), IME (Biomedicina) e ISOC (Ciencias Sociales y Humanidades), que han venido recogiendo desde la década de 1970 la bibliografía de las publicaciones científicas españolas con análisis de contenido. Durante los últimos años se ha rediseñado este sistema de información, lo que hacía recomendable esperar que finalizara el trabajo de exportación al nuevo sistema para manejar datos ya revisados.

El objetivo de este artículo es caracterizar el contenido actual de las revistas seleccionadas con un doble propósito: a) facilitar a los potenciales lectores una información que pueda orientar sus opciones de consulta y publicación y b) contribuir a la Historia de la ciencia, definiendo las pautas de difusión científica de los arqueólogos orientados hacia la Prehistoria y/o hacia la Historia antigua en la última década.

\section{PRESENCIA EN BASES DE DATOS Y PLATAFORMAS DE REVISTAS}

La tabla 1 resume la presencia de $T P$ en bases de datos y plataformas de revistas científicas, especificando sus diferencias en la cobertura temporal de sus artículos.

La revista en versión impresa está disponible en numerosas bibliotecas universitarias en todo el mundo, pero los usuarios demandan hoy en día el acceso telemático a los textos en su versión electrónica. La fuente principal a la versión en texto completo y en abierto es la plataforma de la Editorial CSIC (http:// revistas.csic.es). Esta iniciativa, iniciada en 2007, de momento cubre desde el volumen 45 de 1988. Para la consulta de los primeros años en formato digital es necesario acudir a un producto comercial: PIO-Periodicals Index Online, base de datos bibliográfica, comercializada por la empresa ProQuest, que recoge el fondo antiguo de publicaciones periódicas de Huma-

\begin{tabular}{|l|l|l|}
\hline $\begin{array}{l}\text { Base de datos o } \\
\text { Plataforma }\end{array}$ & Cobertura & Características \\
\hline Revistas CSIC & $1988-2018$ & $\begin{array}{l}\text { TC (plataforma } \\
\text { editorial) }\end{array}$ \\
\hline $\begin{array}{l}\text { PIO Periodicals Index } \\
\text { Online-PAO } \\
\text { Periodicals Archive } \\
\text { Online }\end{array}$ & $1960-2000$ & $\begin{array}{l}\text { TC (fondo } \\
\text { histórico) }\end{array}$ \\
\hline ÍnDICEs-CSIC & $1960-2018$ & $\begin{array}{l}\text { REF, } \\
\text { multidisciplinar }\end{array}$ \\
\hline Dialnet & $1970-2018$ & $\begin{array}{l}\text { REF, } \\
\text { multidisciplinar }\end{array}$ \\
\hline REDIB & $1988-2018$ & $\begin{array}{l}\text { REF, } \\
\text { multidisciplinar }\end{array}$ \\
\hline DOAJ & $1988-2018$ & $\begin{array}{l}\text { REF, } \\
\text { multidisciplinar }\end{array}$ \\
\hline $\begin{array}{l}\text { Anthropological } \\
\text { Literature }\end{array}$ & $1969-2018$ & $\begin{array}{l}\text { REF, especializada } \\
\text { en Antropología }\end{array}$ \\
\hline $\begin{array}{l}\text { Web of Science (Arts } \\
\text { \& Humanities Citation } \\
\text { Index y Social Science } \\
\text { Citation Index) }\end{array}$ & $2007-2018$ & Índice de citas \\
\hline Scopus & $2003-2018$ & Índice de citas \\
\hline
\end{tabular}

Tab. 1. Cobertura en bases de datos bibliográficas y plataformas editoriales de la revista Trabajos de Prehistoria. TC texto completo; REF referencias bibliográficas con enlaces al texto completo.

nidades y Ciencias Sociales. Cuenta con un fichero paralelo, PAO-Periodicals Archive Online, para los artículos que han sido digitalizados.

El contenido de $T P$ es analizado de forma sistemática por buscadores académicos y bases de datos bibliográficas, aunque también con coberturas variables (Tab. 1). A través de estos sistemas de información, los artículos pueden aparecer en búsquedas en portales que recogen la producción española (ÍnDICEs-CSIC https://indices.csic.es/) o hispánica (Dialnet https:// dialnet.unirioja.es), en recolectores de datos de revistas electrónicas (REDIB-Red Iberoamericana de Innovación y Conocimiento Científico https://www.redib.org; DOAJ-Directory of Open Access Journals https://doaj. org/) y en productos bibliográficos especializados (Anthropological Literature).

La revista forma parte también de la selección de fuentes utilizada en los índices de citas WoS y Scopus. En la plataforma $W o S$ aparece tanto en la base de datos Arts \& Humanities Citation Index (en la categoría Archaeology), como en Social Science Citation Index (categoría Anthropology). Su productor no incluye datos estadísticos de factor de impacto $u$ otros indicadores para las revistas de Humanidades en sus informes Journal Citation Reports (JCR), por lo que 
$T P$ solo tiene indicadores de citación entre las de Antropología. Dentro de la categoría de Arqueología sí podemos contar con las fuentes bibliométricas basadas en los datos de Scopus. Estos datos se analizan más adelante.

En la valoración de características editoriales de la red iberoamericana Latindex (http://latindex.org/), TP cumple en 2018 la totalidad de los 33 criterios de calidad en la edición impresa y 35 sobre 36 en la versión electrónica ${ }^{1}$, por lo que en ambos casos entra en la sección de Catálogo. Las características de calidad definidas por esta red iberoamericana, coordinada desde la Universidad Nacional Autónoma de México, valoran el cumplimiento de buenas prácticas en los procesos de edición científica.

La propia presencia en bases de datos se utiliza a menudo como indicador de la calidad o prestigio de las revistas científicas. Entre las fuentes que valoran especialmente este indicador, $T P$ se sitúa en la categoría A en Ciencias Humanas en CIRC (Clasificación Integrada de Revistas Científicas: https://www.clasificacioncirc.es/) y tiene un Índice Compuesto de Difusión Secundaria (ICDS) con el nivel máximo de 11 en la plataforma MIAR (Matriz de Información para el Análisis de Revistas: http://miar.ub.edu). Otro sistema de categorización de revistas en el que ha sido seleccionada es ERIH Plus, European Reference Index for Humanities and Social Sciences (https://dbh.nsd.uib.no/publiseringskanaler/erihplus/), una iniciativa de la European Science Foundation para crear un índice de revistas académicas europeas de calidad en Ciencias Sociales y Humanidades.

TP cuenta desde 2012 con el Sello de Calidad de Revistas Científicas Españolas concedido por la Fundación Española para la Ciencia y la Tecnología (FECYT). Este sello, que se ha ido renovando, certifica su posición entre las revistas científicas españolas de excelencia por su difusión internacional y cumplimiento de buenas prácticas de edición y utilización de revisores externos ${ }^{2}$. En la categorización de las revistas españolas de Arqueología y Prehistoria, realizada en 2015 en la base de datos ISOC a partir de indicadores sobre trayectoria, apertura de la autoría e internacionalidad en el periodo 2004-2013, TP quedó encuadrada en la categoría B (Vidal Liy y Rodríguez Yunta 2015). De 9 indicadores, mejoraba la media de la disciplina en 8 de ellos.

\footnotetext{
${ }^{1}$ De los criterios marcados por Latindex para revistas electrónicas TP solamente dejaba de cumplir en 2018 la presencia de metaetiquetas en el código fuente de los artículos. En 2019 se está aplicando una nueva lista de características de calidad y la revista está en proceso de adaptación a su cumplimiento.

${ }^{2}$ En 2019 la FECYT ha elaborado un ranquin de las revistas con este sello de calidad, donde TP es la publicación de Arqueología y Prehistoria con mejor puntuación. https://calidadrevistas.fecyt.es/
}

\section{METODOLOGÍA}

Los datos para el análisis bibliométrico de $T P$ se han extraído del recurso ya citado ÍnDICEs-CSIC. Este portal bibliográfico incorpora los registros antes disponibles en la base ISOC y ofrece una cobertura completa de la revista (1960-2018) con campos para el análisis de autoría, filiación institucional, clasificación e indización de los temas tratados en cada artículo (asignando descriptores de materias, identificadores y topónimos). La base de datos ISOC ha sido fuente para el primer estudio sobre TP (Rodríguez Alcalde et al. 1993), así como sobre otras revistas de la disciplina (García del Toro et al. 1999).

Un aspecto novedoso del análisis actual es la comparación respecto a otras revistas españolas especializadas en Arqueología a la vez que a estas entre sí. Para ello se han seleccionado títulos incluidos también en ÍnDICEs-CSIC y en Scopus con los que compartiera cierta similitud de enfoque (cobertura temporal y temática de los artículos) (Tab. 2) ${ }^{3}$. Se excluyen de la comparativa títulos españoles ausentes de Scopus y otros presentes pero con enfoques temáticos muy específicos (Archaeofauna, Arqueología de la Arquitectura o Virtual Archaeology Review) o ajenos a la Prehistoria de la península ibérica (Arqueología Iberoamericana, Aula Orientalis).

La mayoría de las revistas seleccionadas tiene una extensa trayectoria ${ }^{4} \mathrm{y}$ aceptan textos en diferentes idiomas $^{5}$. TP fue la primera en adoptar una periodicidad semestral (1994), ejemplo que han seguido posteriormente cuatro títulos. Todas tienen la particularidad de que la entidad editora es una institución pública, mientras que el panorama internacional está dominado por revistas comerciales: 7 de las 10 primeras revistas en el ranquin por factor de impacto de la categoría Anthropology en $\mathrm{WoS}$ e igualmente por el indicador CiteScore de la categoría Archeology en Scopus, pertenecen a las poderosas empresas multinacionales Elsevier, Springer Nature, Wiley-Blackwell, Taylor \& Francis y SAGE.

Todas las revistas actualmente tienen versión electrónica. En este proceso las revistas del CSIC y de la

\footnotetext{
3 En los títulos de algunas de las revistas seleccionados los editores utilizan en ocasiones la grafía latina $\mathrm{V}$ para el sonido $\mathrm{U}$ (Lvcentvm, Sagvntvm, Zephyrvs). En este artículo se ha normalizado la denominación a la grafía con $U$.

${ }^{4}$ Algunas cuentan con antecedentes de títulos anteriores, que si se incorporan aumentan su longevidad. Archivo Español de Arqueología puede considerarse un desdoblamiento de título de Archivo Español de Arte y Arqueología (1925-1937). Munibe. Antropologia-Arkeologia (en adelante Munibe A-A) es un cambio de título de Munibe. Antropología y Arqueología, que a su vez surge en 1984 al desdoblarse el título $M u$ nibe, iniciado en 1949.

5 Dato extraído de las normas para autores publicadas por cada revista en su sitio web.
}

Trab. Prehist., 76, N. ${ }^{\circ}$ 2, julio-diciembre 2019, pp. 199-218, ISSN: 0082-5638

https://doi.org/10.3989/tp.2019.12233 


\begin{tabular}{|l|c|c|c|c|c|c|}
\hline Revista & $\begin{array}{c}\text { Entidad } \\
\text { editora }\end{array}$ & $\begin{array}{c}\text { Año de } \\
\text { inicio }\end{array}$ & $\begin{array}{c}\text { Perio- } \\
\text { dicidad }\end{array}$ & Lenguas de publicación & $\begin{array}{c}\text { Cobertura en } \\
\text { WoS }\end{array}$ & $\begin{array}{c}\text { Cobertura en } \\
\text { Scopus }\end{array}$ \\
\hline AEspA & CSIC & $1940(1925)$ & Anual & No se especifica & $2007-$ & $2009-$ \\
\hline APL & SIP & 1928 & Bienal & ES, CA, EN, FR, IT & No & $2010-$ \\
\hline Complutum & UCM & 1991 & Sem. 2008- & ES, EN & $2015-$ & $2011-$ \\
\hline Lucentum & UA & 1982 & Anual & No se especifica & $2014-$ & $2015-$ \\
\hline Munibe A-A & SCA & $1987(1949)$ & Anual & EU, ES, FR, EN & Irregular & $2013-$ \\
\hline Pyrenae & UB & 1965 & Sem. 2004- & CA, ES, EN & No & $2015-$ \\
\hline Saguntum & UV & 1962 & Anual & ES, CA, PO, FR, IT, EN, DE & No & $2017-$ \\
\hline Spal & US & 1993 & Sem. 2018- & ES, EN, FR, IT, PO, DE & $2015-$ & $2015-$ \\
\hline TP & CSIC & 1960 & Sem. 1994- & ES, EN, FR & $2007-$ & $2003-$ \\
\hline Zephyrus & USAL & 1950 & Sem. 2008- & ES, EN, FR & $2015-$ & $2011-$ \\
\hline
\end{tabular}

Tab. 2. Revistas españolas que se comparan en el estudio, entidad editora, año de inicio, idiomas de publicación y cobertura en índices de citas internacionales. Sem. Semestral, CA catalán, DE alemán, EN inglés, ES español, EU euskera, FR francés, IT italiano, PO portugués. Las abreviaturas de instituciones editoras se especifican al final del artículo.

UCM fueron las primeras en registrar un ISSN para la versión de Internet en 2007. Un apartado especial merece el tratamiento del sistema de evaluación de manuscritos empleado por cada revista. En todos los casos figura en las normas la utilización de al menos dos evaluadores externos. En AEspA, Complutum, Munibe, Pyrenae, Saguntum y Zephyrus se especifica el empleo del sistema doble ciego; $T P$ utiliza en la actualidad el simple ciego. Solamente las dos revistas de Editorial CSIC publican en su sitio web cada tres o cuatro años la lista de evaluadores (excluyendo los que no lo hayan autorizado).

El análisis de temática y autoría de $T P$ se basa en búsquedas en febrero de 2019 en el total de 933 registros de la revista acumulados en ÍnDICEs-CSIC desde su inicio en 1960. El total engloba 603 contribuciones catalogadas como artículos (sección de estudios), 295 notas (sección de noticiario), 18 revisiones o estados de la cuestión, 12 traducciones. Se han excluido necrológicas, reseñas, crónicas y editoriales.

El sistema de clasificación temática de ÍnDICEsCSIC es la base del estudio de los contenidos tratados en la trayectoria de TP (1960-2018). Aunque un mismo registro puede tener asignados varios epígrafes de clasificación, para este análisis se ha manejado el primero, que describe el enfoque considerado principal en cada artículo. A su vez, a partir de los topónimos que citan se define la distribución territorial de los temas tratados.

Para la autoría se contabiliza el número de firmantes en los artículos y su filiación institucional. Generalmente estos datos provienen de los propios artículos aunque, como no solía figurar en los primeros años, se ha completado en un número significativo de casos, a fin de aportar una visión más objetiva y fiable.
La comparación con otras revistas se ha centrado en el periodo 2010-2018. Además de mostrar su situación actual, permite confrontar unas con otras en condiciones idénticas de cobertura, dado que su fecha de inicio varía. Los datos sobre citación se han extraído de Scopus. Su cobertura es algo mayor que la de WoS y permite la comparación con un mayor número de títulos españoles. Se ha desestimado utilizar datos de citas del buscador académico Google Scholar por considerarlo carente de la estabilidad y fiabilidad estadística que aportan las bases de datos bibliográficas.

\section{ANÁLISIS DEL CONTENIDO DE LOS ARTÍCULOS}

\subsection{Análisis por periodos y temas tratados en $T P$}

El análisis de contenido de los 933 artículos y notas de investigación de TP (1960-2018) sigue la asignación principal de cada registro en el área de Arqueología y Prehistoria de la base de datos ÍnDICEs-CSIC (Fig. 1). Su primer nivel de clasificación temática incluye epígrafes de carácter general: Teoría y metodología de la Arqueología (teoría, historia, enseñanza y metodología de la Arqueología), Patrimonio arqueológico (museos, conservación y legislación), Origen de la humanidad y Estudios generales (trabajos diacrónicos). Además, hay otros relativos referidos a la Prehistoria y Arqueología de la península ibérica y otros territorios (estudios sobre las islas Canarias, Europa, Asia, África y América). Los epígrafes relativos a la península ibérica se dividen por periodos cronológicos: Paleolítico y Epipaleolítico; Neolítico; Calcolítico y Edad del Bronce; Tarteso y colonizaciones griega y 


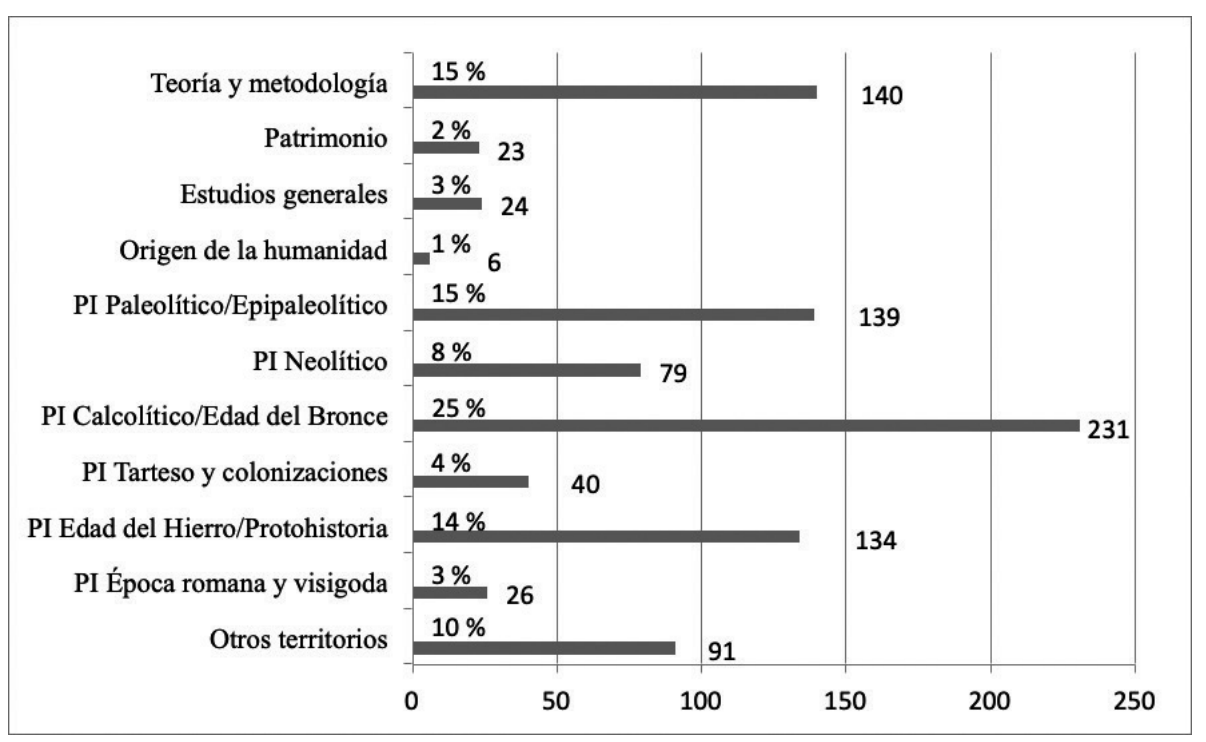

Fig. 1. Cantidad y porcentaje del total de 933 artículos de Trabajos de Prehistoria (1960-2018) según la clasificación temática en ÍnDICEs CSIC. PI península ibérica. La categoría Estudios generales incluye estudios diacrónicos; Otros territorios engloba los estudios sobre las islas Canarias, Europa, Asia, África y América; Teoría y metodología agrupa a la teoría, historia, enseñanza y metodología arqueológicas.

fenopúnica; Edad del Hierro; Época romana y visigoda y Edad Media. Estos epígrafes reducen los existentes en la base de datos ISOC cuando la manejaron Rodríguez Alcalde et al. (1993), agrupando en especial los referidos a periodos cronológicos que solían solaparse en los mismos trabajos.

Los estudios referidos a la teoría y metodología arqueológicas tienen una presencia modesta en $T P$ (15\%). Sin embargo, tuvieron mayor peso en la década de 1990 con 66 artículos (32,5\% del total de dicho periodo). El interés por los temas patrimoniales ha sido aún menor. Su momento álgido corresponde a la década de 2000 con 14 artículos (7\% en dicho periodo).

En la distribución de los 649 artículos referidos a la península ibérica (Tab. 3) ${ }^{6}$, destaca la coherencia entre la línea editorial de la revista y su contenido. El $69,2 \%$ de estos artículos trata temas anteriores al I milenio a. C., dedicándose casi por igual a los periodos con tecnologías de base cobre $(35,6 \%)$ y no metálicas $(33,6 \%)$. La suma de los correspondientes a los epígrafes del periodo de contacto con pueblos históricos, previos a la romanización, no alcanza esos valores $(26,8 \%)$.

La misma estructura por periodos cronológicos se repite en el estudio por décadas de la revista desde

\footnotetext{
${ }^{6}$ Esta cifra se limita a los documentos clasificados en los epígrafes de ÍnDICEs CSIC sobre Prehistoria y Arqueología de la península ibérica. Se dejan fuera de este análisis los 6 artículos sobre Origen de la humanidad y los 24 referidos a Estudios generales, ya que en ambos casos puede tratarse de otros territorios.
}

1970 (Tab. 4) $^{7}$. Las contribuciones sobre temáticas previas al I milenio a. C. son mayoritarias en todos los casos (media 68,8\%), aumentando desde 1990. Las medias relativas al Calcolítico y la Edad del Bronce $(35,4 \%)$ y a los periodos previos $(33,4 \%)$ ocultan diferencias por décadas entre ambas agrupaciones de artículos y en la composición interna de la segunda. Los dedicados al Paleolítico/Epipaleolítico son el $16,8 \%$ por término medio en cada una, descendiendo entre 1980 y 1999. Los de Neolítico son el $12,2 \%$ de media superándola desde 1990, cuando por primera vez en los 48 años, incluso hay un artículo más que los relativos a los periodos previos. A su vez la media de los clasificados en el Calcolítico y la Edad del Bronce varía de 27 a $38 \%$ en décadas alternantes.

Los artículos del periodo de contacto con pueblos históricos, previos a la romanización, son el 26,8 \% de media por década y los dedicados a la Edad del Hierro $(20,8 \%)$ duplican o triplican siempre a los de Tarteso y colonizaciones (media $6 \%$ ). La trayectoria de estos últimos es oscilante hasta el 2000 cuando descienden y se estabilizan. La proporción de artículos sobre la Edad de Hierro es inferior a la media en la primera y última década y superior entre 1980-2009. Por último, los estudios sobre la Época romana y vi-

\footnotetext{
${ }^{7}$ La de 1960-1969 queda excluida porque los números eran monográficos y a veces referidos a Otros territorios. En total se publicaron 28 artículos dedicados a las comunidades autónomas o a España (Rodríguez Alcalde et al. 1993: 22, tab. 3).
} 


\begin{tabular}{|c|c|c|c|c|c|c|c|c|c|c|}
\hline \multirow{2}{*}{ Periodo } & \multicolumn{2}{|c|}{ 1970-1979 } & \multicolumn{2}{|c|}{ 1980-1989 } & \multicolumn{2}{|c|}{ 1990-1999 } & \multicolumn{2}{|c|}{ 2000-2009 } & \multicolumn{2}{|c|}{ 2010-2018 } \\
\hline & Art. & $\%$ & Art. & $\%$ & Art. & $\%$ & Art. & $\%$ & Art. & $\%$ \\
\hline Paleolítico/Epipaleolítico & 28 & 24 & 17 & 17 & 17 & 15 & 37 & 26 & 38 & 24 \\
\hline Neolítico & 9 & 8 & 6 & 6 & 18 & 16 & 21 & 15 & 26 & 16 \\
\hline Calcolítico/Edad del Bronce & 40 & 34 & 42 & 42 & 40 & 36 & 38 & 27 & 60 & 38 \\
\hline Tarteso y colonizaciones & 8 & 7 & 5 & 5 & 11 & 10 & 6 & 4 & 6 & 4 \\
\hline Edad del Hierro & 16 & 14 & 27 & 27 & 24 & 21 & 36 & 26 & 26 & 16 \\
\hline Época romana y visigoda & 15 & 13 & 3 & 3 & 2 & 2 & 2 & 1 & 2 & 1 \\
\hline Total artículos sobre PI & 116 & 100 & 100 & 100 & 112 & 100 & 140 & 100 & 158 & 100 \\
\hline
\end{tabular}

Tab. 4. Distribución por décadas desde 1970 de los artículos referidos a periodos de la península ibérica en Trabajos de Prehistoria. Art artículos. PI península ibérica.

sigoda pasan del $13 \%$ en la primera década al 3 y $1 \%$ a partir de 1980 .

Sobre la base de nuestra experiencia en la edición de Trabajos de Prehistoria conectamos estos datos con dos factores que se refuerzan y contraponen en cada número. El primero es la voluntad del consejo de redacción de publicar en cada número contribuciones representativas de la línea editorial. El segundo es el carácter abierto de la recepción de manuscritos. Su envío a la revista depende de la estrategia de los autores para difundir su investigación y de la disponibilidad de una información inédita que suele estar subordinada a la importancia de los recursos con los que han contado.

El sistema de clasificación temática de ÍnDICEsCSIC completa el análisis de los estudios sobre la península ibérica con un segundo nivel de 8 apartados (Tab. 5): Asentamientos, Estudios del medio, Economía y sociedad, Enterramientos, Religión y creencias, Arte, Cultura material (que aúna a las industrias lítica, ósea y metalúrgica y a la producción cerámica) y Epigrafía/ Numismática.

\begin{tabular}{|l|c|c|}
\hline Periodo & Núm. arts. & $\mathbf{\%}$ \\
\hline Asentamientos & 86 & 13,3 \\
\hline Estudios del medio & 44 & 6,8 \\
\hline Economía y sociedad & 52 & 8 \\
\hline Enterramientos & 96 & 14,8 \\
\hline Religión y creencias & 16 & 2,5 \\
\hline Arte & 108 & 16,6 \\
\hline Cultura material & 242 & 37,3 \\
\hline Epigrafía y numismática & 5 & 0,8 \\
\hline $\begin{array}{l}\text { Total de artículos sobre } \\
\text { península ibérica }\end{array}$ & 649 & 100 \\
\hline
\end{tabular}

Tab. 5. Cantidad y porcentaje por temas del total de artículos referidos a la península ibérica en Trabajos de Prehistoria (1960-2018).
Las categorías Cultura material (242 artículos) y Arte (108) concentran la mayor parte de los artículos (53,9 \% entre ambas). La primera aparece en los estudios relativos a todos los periodos, desde el Paleolítico a la época romana. Por el contrario, los trabajos sobre Arte se concentran en el Paleolítico y Edad del Bronce. Enterramientos es la categoría donde la Edad del Bronce supera con mayor claridad a los demás períodos $(68,4 \%$ de los trabajos $)$.

\subsection{Análisis por áreas geográficas en $T P$}

El objetivo de esta sección del artículo es averiguar el ámbito territorial al que se dedican los autores que envían sus contribuciones a TP. En su mayoría se centran en la península ibérica y en los archipiélagos balear y canario. Solamente 89 artículos $(9,5 \%)$ tratan temas extrapeninsulares de Europa (45), África (25), América (14), Asia y Oriente Próximo (5). Estos estudios $\mathrm{y}$, sobre todo, los de territorios no europeos van disminuyendo durante los 48 años estudiados a medida que $T P$ se va consolidando como revista de referencia para la Prehistoria y Protohistoria del extremo más occidental del Mediterráneo.

El análisis de la distribución territorial de los artículos sobre la península ibérica se hará a escala autonómica (Tab. 6) y, después, limitado a las provincias mejor representadas y a Portugal (Fig. 2). En ambos casos se considera la totalidad de los artículos. Después se comparan los estudios por comunidades autónomas entre 1960-2009 y 2010-2018.

El primer dato que se desprende del análisis es el interés de $T P$ por la Prehistoria y Protohistoria de la península ibérica en su conjunto. Todas las comunidades autónomas están representadas con independencia de su extensión. Andalucía es ligeramente menor que Castilla y León, pero la diferencia del total de artículos entre ellas es notable $(21,1 \%$ y $12 \%)$. La misma desconexión 


\begin{tabular}{|l|c|c|c|c|c|c|c|}
\hline \multirow{2}{*}{ Comunidad autónoma } & \multirow{2}{*}{$\mathbf{\% ~ k m}^{2}$} & \multicolumn{2}{|c|}{ Total } & \multicolumn{2}{c|}{$\mathbf{1 9 6 0 - 2 0 0 9}$} & \multicolumn{2}{c|}{$\mathbf{2 0 1 0 - 2 0 1 8}$} \\
\cline { 3 - 8 } & & Artículos & $\mathbf{\%}$ & Artículos & $\mathbf{\%}$ & Artículos & $\mathbf{\%}$ \\
\hline Castilla y León & 18,6 & 85 & 12,0 & 70 & 12,6 & 15 & 9,7 \\
\hline Andalucía & 17,3 & 149 & 21,1 & 126 & 22,8 & 23 & 14,9 \\
\hline Castilla-La Mancha & 15,7 & 52 & 7,3 & 33 & 6,0 & 19 & 12,3 \\
\hline Aragón & 9,4 & 14 & 2,0 & 8 & 1,4 & 6 & 3,9 \\
\hline Extremadura & 8,2 & 44 & 6,2 & 33 & 6,0 & 11 & 7,1 \\
\hline Cataluña & 6,3 & 71 & 10,0 & 44 & 7,9 & 27 & 17,5 \\
\hline Galicia & 5,8 & 46 & 6,5 & 35 & 6,3 & 11 & 7,1 \\
\hline Comunidad Valenciana & 4,6 & 51 & 7,2 & 44 & 7,9 & 7 & 4,5 \\
\hline Región de Murcia & 2,2 & 27 & 3,8 & 22 & 4,0 & 5 & 3,2 \\
\hline Navarra & 2,1 & 3 & 0,4 & 3 & 0,5 & 0 & 0,0 \\
\hline Principado de Asturias & 2,0 & 30 & 4,2 & 27 & 4,9 & 3 & 1,9 \\
\hline Comunidad de Madrid & 1,6 & 24 & 3,4 & 17 & 3,0 & 7 & 4,5 \\
\hline Canarias & 1,5 & 10 & 1,4 & 9 & 1,6 & 1 & 0,6 \\
\hline País Vasco & 1,4 & 14 & 2,0 & 9 & 1,6 & 5 & 3,2 \\
\hline Cantabria & 1,1 & 36 & 5,1 & 30 & 5,4 & 6 & 3,9 \\
\hline La Rioja & 1,0 & 3 & 0,4 & 2 & 0,4 & 1 & 0,6 \\
\hline Illes Balears & 1,0 & 48 & 6,8 & 41 & 7,4 & 7 & 4,5 \\
\hline Total artículos sobre CC.AA & & 707 & 100 & 553 & 100 & 154 & 100 \\
\hline
\end{tabular}

Tab. 6. Comparación de las comunidades autónomas representadas en Trabajos de Prehistoria en su conjunto, en el periodo 1960-2009 y en la última década 2010-2018. Las comunidades se ordenan por su mayor o menor superficie respecto a la de España. El porcentaje está calculado sumando los artículos que tratan sobre comunidades autónomas específicas.

ocurre con las siguientes en el ranquin. Cataluña $(6,3 \%$ $\left.\mathrm{km}^{2} ; 10 \%\right)$, Castilla la Mancha $\left(15,7 \% \mathrm{~km}^{2} ; 7,3 \%\right) \mathrm{y}$ la Comunidad Valenciana $(4,6 \%-7,2 \%)$.

La situación a escala provincial permite afinar más estos datos (Fig. 2). Todas las provincias españolas y Portugal cuentan con artículos, pero hay notables di-

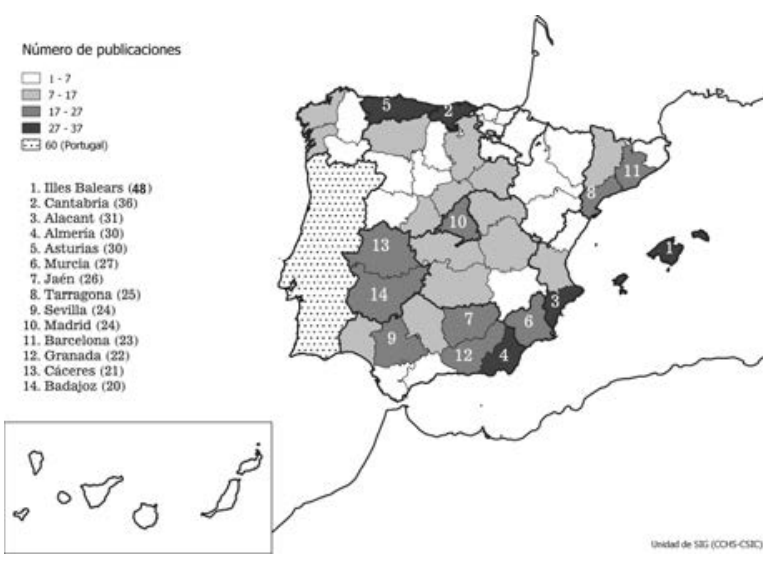

Fig. 2. Representación de Portugal y de las provincias españolas en los artículos de Trabajos de Prehistoria (1960-2018). ferencias entre ellas. El señalado protagonismo de Andalucía se debe al interés de los autores por registros arqueológicos localizados en Almería, Jaén, Sevilla y Granada (más de 20 artículos cada una). A su vez la atención en Castilla y León se dirige a las cinco provincias que superan las 7 contribuciones en ese periodo (Ávila, Burgos, León, Segovia y Soria) y, en Cataluña, a Tarragona y Barcelona (24 de media). La presencia de Castilla-La Mancha en el ranquin se debe a una distribución homogénea por provincias (entre 7-17), de la que solo Albacete queda fuera. En la Comunidad Valenciana cada una está en un tramo por número de artículos situándose Alacant en el más alto. En cambio, en general, no han recurrido a $T P$ para difundir su investigación ni los estudiosos del País Vasco y de Aragón (ninguna de las provincias cuenta con más de 7), ni menos todavía los de Navarra y La Rioja (3 artículos cada una). Como contrapunto las comunidades uniprovinciales de Illes Balears, Cantabria, Asturias, Murcia y Madrid están bien situadas entre las 14 provincias con más contribuciones publicadas en la revista (entre 48 y 24).

Las explicaciones de esta "foto fija" de la distribución territorial en los 48 años analizados de publicación

Trab. Prehist., 76, N. ${ }^{\circ}$ 2, julio-diciembre 2019, pp. 199-218, ISSN: 0082-5638

https://doi.org/10.3989/tp.2019.12233 
de $T P$ no difieren de las ya apuntadas (RodríguezAlcalde et al. 1993: 21-22). Entre ellas están la primacía de unos períodos o de unas culturas particulares, el número de centro académicos, las afiliaciones institucionales de los autores, la facilidad de acceso a los permisos de excavación y a la financiación, la procedencia del alumnado universitario y el papel de las revistas especializadas de ámbito local, provincial o autonómico.

El factor temporal contribuye a dar un nuevo sentido a los datos. Entre 1960 y 2009 predominan los artículos sobre Andalucía (22,8 \%) y Castilla y León $(12,6 \%)$, seguidos por los dedicados a Cataluña, Comunidad Valenciana $(7,9 \%)$ y Baleares $(7,4 \%)$. En la última década 2010-2018 es notable el cambio del ámbito territorial de quienes se interesan por publicar en $T P$. Cataluña $(17,5 \%)$, Andalucía $(14,9 \%)$ y Castilla la Mancha $(12,3 \%)$ son las comunidades mejor representadas en los artículos. En cambio, Castilla y León pasa a un cuarto lugar $(9,7 \%)$ y a continuación aparecen Extremadura y Galicia (7,1 \%). Una consecuencia de centrar la línea editorial de la revista en la península ibérica es el interés por incorporar trabajos sobre Portugal que cuenta con 60 artículos. Este país ha estado presente en las primeras décadas de la revista (5 en la de 1960, 13 en la de 1970, 12 en la de 1980, 9 en la de 1990 y 6 en la de 2000), con un significativo crecimiento en la última (15 artículos en 2010-2018).

La precariedad del marco institucional y la escasa planificación de la actividad arqueológica centralizada van superándose a lo largo de las primeras décadas del periodo estudiado a medida que se consolida la administración autonómica y la incorporación a la UE. Por poner un ejemplo, de 1987 (creación del programa Erasmus) a 1998, se crean 19 universidades públicas en España que, salvo dos, tienen cursos de arqueología. La competencia entre investigadores se acrecienta y los criterios de evaluación se internacionalizan, modificando las estrategias de difusión científica y selección de revistas. Como contrapunto las comunidades autónomas a las que la administración central del Estado ha trasferido las competencias en arqueología suelen reservarse la primera publicación de los resultados de las intervenciones. En su defecto o con el debido permiso se opta por las revistas editadas en la comunidad o en España. Esto dota de ventajas competitivas a las de ámbito peninsular como $T P$.

\subsection{Análisis del contenido en comparación con otras revistas}

La tabla 7 compara con otras revistas la distribución temática de los artículos entre 2010 y 2018. La proporción de artículos por periodos en cada título permite distinguir tres líneas editoriales. Una, orientada hacia la arqueología romana, está representada por AEspA y Lucentum, con más de la mitad o la mitad de sus artículos sobre el tema, y Spal y Pyrenae, con algo más de un tercio. AEspA y Lucentum se interesan también por los periodos posteriores al I milenio a. C. $y$, en especial, por la Edad del Hierro lo que refuerza los contenidos de Protohistoria e Historia Antigua de sus artículos.

Zephyrus tiene también un interés análogo por la época romana (32\%). Sin embargo, a diferencia de las citadas, dedica la mitad de su contenido a los periodos previos al I milenio a. C. Por ello pensamos que encaja mejor en la segunda línea editorial, definida por $A P L$, Munibe y $T P$ donde esos temas rondan o superan el $50 \%$. Los periodos premetalúrgicos tienen un especial tratamiento en $A P L$, Munibe, Zephyrus y $T P$, si bien en esta última lo distintivo o exclusivo es su orientación hacia el Calcolítico y la Edad del Bronce $(31,6 \%$ de artículos),

En $A P L$ el alto porcentaje de artículos sobre el Neolítico ("cardial") y la Edad del Hierro ("ibérico") se relaciona con el protagonismo tradicional del Levante peninsular en estos estudios. En TP los referidos al Calcolítico/Edad del Bronce reflejan una tradición investigadora bien arraigada (Rovira Llorens y Montero Ruíz 2018). Munibe se singulariza en el conjunto de revistas por carecer de artículos sobre Tarteso y colonizaciones, contar con el mínimo de los dedicados a la Edad del Hierro $(6,4 \%)$ y, en cambio, tener el máximo de contribuciones sobre Edad Media (9,6\%) y Moderna $(7,7 \%)$.

Complutum define una tercera línea editorial en esta selección de revistas y se caracteriza por un enfoque mayoritario hacia la metodología y patrimonio arqueológicos $(41,2 \%)$. En los artículos por periodos, los relativos a periodos previos $(18,4 \%)$ y posteriores al I milenio (15,2\%) están bastante igualados. Esa proporcionalidad se debe a los contenidos sobre la Edad del Hierro. La revista comparte con TP la ausencia de contribuciones sobre la Edad Media o Moderna.

En la tabla 8 se compara la distribución por comunidades autónomas tratadas en los artículos. Destaca el contraste entre las revistas con sede en Madrid AEspA, Complutum y $T P-$ y fuera de la capital. Los contenidos de las primeras pueden referirse a unas comunidades más que a otras, pero tienden a abarcar toda la península, mientras las que tienen sede en otras regiones centran al menos un tercio de los artículos en su propio territorio.

Respecto a la presencia de Portugal en las revistas españolas comparadas, solamente Spal iguala el número de artículos en TP: 15 en 2010-2018. A conti- 


\begin{tabular}{|c|c|c|c|c|c|c|c|c|c|c|c|c|c|c|}
\hline Revistas & $\mathbf{P} / \mathbf{E}$ & $\mathbf{N}$ & $\mathrm{C} / \mathrm{EBr}$ & $\mathrm{T} / \mathrm{C}$ & EH & Roma & EMed & EMod & TM & Patr & OT & EG & $\mathrm{OH}$ & Total \\
\hline AEspA & 0 & 0 & $2(1,5)$ & $11(8,2)$ & $19(14,2)$ & $74(55,2)$ & $8(6,0)$ & 0 & $8(6,0)$ & $1(0,7)$ & $10(7,5)$ & $1(0,7)$ & 0 & 134 \\
\hline$A P L$ & $18(24,3)$ & $10(13,5)$ & $3(4,0)$ & $2(2,7)$ & $14(18,9)$ & $12(16,2)$ & $4(5,4)$ & $1(1,3)$ & $3(4,0)$ & $4(5,4)$ & $1(1,7)$ & $2(2,7)$ & 0 & 74 \\
\hline Complutum & $8(3,8)$ & $9(4,3)$ & $24(11,4)$ & $6(2,8)$ & $31(14,7)$ & $6(2,8)$ & 0 & 0 & $50(23,7)$ & $37(17,5)$ & $36(17,0)$ & $3(1,4)$ & $1(0,5)$ & 211 \\
\hline Lucentum & $3(2,3)$ & 0 & $5(3,8)$ & $9(6,8)$ & $20(15,1)$ & $67(50,7)$ & $5(3,8)$ & $1(0,7)$ & $4(3,0)$ & $6(4,5)$ & $11(8,3)$ & $1(0,7)$ & 0 & 132 \\
\hline Munibe & $52(29,5)$ & $19(10,8)$ & $19(10,8)$ & 0 & $11(6,2)$ & $19(10,8)$ & $19(10,8)$ & $12(6,8)$ & $6(3,4)$ & $1(0,6)$ & $13(7,4)$ & $3(1,7)$ & $1(0,6)$ & 176 \\
\hline Pyrenae & $7(6,6)$ & $3(2,8)$ & $2(1,9)$ & $2(1,9)$ & $10(9,4)$ & $36(34,0)$ & $8(7,5)$ & 0 & $21(19,8)$ & $3(2,8)$ & $13(12,3)$ & 0 & $1(0,9)$ & 106 \\
\hline Saguntum & $15(5,5)$ & $10(3,6)$ & $16(5,8)$ & $13(4,7)$ & $49(17,9)$ & $58(21,2)$ & $12(4,4)$ & $5(1,8)$ & $40(14,6)$ & $4(1,4)$ & $41(15,0)$ & $11(4,0)$ & 0 & 274 \\
\hline Spal & $2(1,7)$ & $5(4,4)$ & $14(12,3)$ & $14(12,3)$ & $13(11,4)$ & $46(40,3)$ & $2(1,7)$ & $1(0,9)$ & $10(8,8)$ & $1(0,9)$ & $6(5,3)$ & 0 & 0 & 114 \\
\hline Zephyrus & $37(20,3)$ & $20(11,0)$ & $34(18,7)$ & $1(0,5)$ & $19(10,4)$ & $58(31,9)$ & $5(2,7)$ & 0 & $1(0,5)$ & $1(0,5)$ & $5(2,7)$ & 0 & $1(0,5)$ & 182 \\
\hline$T P$ & $38(19,9)$ & $26(13,6)$ & $61(31,9)$ & $6(3,1)$ & $26(13,6)$ & $2(1,0)$ & 0 & 0 & $15(7,9)$ & 0 & $15(7,9)$ & $1(0,5)$ & $1(0,5)$ & 191 \\
\hline
\end{tabular}

Tab. 7. Distribución del número de artículos en las revistas españolas de Arqueología incluidas en Scopus entre 2010-2018. Entre paréntesis se indica el porcentaje respecto al total de artículos de cada revista en los años indicados. Los periodos se refieren siempre a la Península Ibérica: P/E Paleolítico y Epipaleolítico; N Neolítico; C/EBr Calcolítico y Edad del Bronce; T/C Tarteso y colonizaciones; EH Edad del Hierro; Roma Arqueología romana; EMed Arqueología medieval; EMod Arqueología moderna e industrial; TM Teoría y metodología arqueológicas; Patr Patrimonio arqueológico; OT Otros territorios (que abarcan Canarias, América, África, Asia y Oceanía); EG Estudios generales y diacrónicos; $\mathrm{OH}$ Origen de la humanidad.

\begin{tabular}{|l|c|c|c|c|c|c|c|c|c|c|}
\hline & AEspA & APL & Complutum & Lucentum & Munibe & Pyrenae & Saguntum & Spal & Zephyrus & TP \\
\hline Andalucía & $28(20,9)$ & $7(9,5)$ & $15(7,1)$ & $23(17,4)$ & $5(2,8)$ & $10(9,4)$ & $12(4,4)$ & $55(48,2)$ & $21(11,5)$ & $23(12)$ \\
\hline Aragón & $7(5,2)$ & $4(5,4)$ & $2(0,9)$ & $1(0,8)$ & $4(2,3)$ & $6(5,7)$ & $5(1,8)$ & 0 & $16(8,8)$ & $6(3,1)$ \\
\hline Asturias & 0 & $1(1,4)$ & $2(0,9)$ & $1(0,8)$ & $16(9,1)$ & $2(1,9)$ & $1(0,4)$ & 0 & $9(4,9)$ & $3(1,6)$ \\
\hline Cantabria & $2(1,5)$ & 0 & $6(2,8)$ & 0 & $20(11,4)$ & $1(0,9)$ & 0 & 0 & $7(3,8)$ & $6(3,1)$ \\
\hline Castilla y León & $11(8,2)$ & 0 & $20(9,5)$ & $7(5,3)$ & $28(15,9)$ & $5(4,7)$ & $3(1,1)$ & $4(3,5)$ & $27(14,8)$ & $15(7,9)$ \\
\hline Castilla-La Mancha & $10(7,5)$ & $7(9,5)$ & $21(10)$ & $24(18,2)$ & $7(4,0)$ & $1(0,9)$ & $14(5,1)$ & $5(4,4)$ & $16(8,8)$ & $19(9,9)$ \\
\hline Cataluña & $15(11,2)$ & $3(4,1)$ & $7(3,3)$ & $7(5,3)$ & $10(5,7)$ & $30(28,3)$ & $22(8)$ & $3(2,6)$ & $5(2,7)$ & $27(14,1)$ \\
\hline Comunidad de Madrid & $1(0,7)$ & 0 & $3(1,4)$ & $2(1,5)$ & $2(1,1)$ & 0 & 0 & 0 & $1(0,5)$ & $7(3,7)$ \\
\hline Comunidad Valenciana & $5(3,7)$ & $48(64,9)$ & $6(2,8)$ & $39(29,5)$ & $3(1,7)$ & $9(8,5)$ & $103(37,6)$ & $2(1,8)$ & $13(7,1)$ & $7(3,7)$ \\
\hline Extremadura & $6(4,5)$ & 0 & $3(1,4)$ & $4(3)$ & $2(1,1)$ & $1(0,9)$ & $7(2,6)$ & $7(6,2)$ & $13(7,1)$ & $11(5,8)$ \\
\hline Galicia & $3(2,2)$ & 0 & $5(2,4)$ & $2(1,5)$ & 0 & $1(0,9)$ & $3(1,1)$ & $1(0,9)$ & $6(3,3)$ & $11(5,8)$ \\
\hline Illes Balears & $5(3,7)$ & 0 & $3(1,4)$ & $2(1,5)$ & $1(0,6)$ & $4(3,8)$ & $14(5,1)$ & $4(3,5)$ & 0 & $7(3,7)$ \\
\hline Islas Canarias & 0 & 0 & $7(3,3)$ & $2(1,5)$ & $1(0,6)$ & 0 & 0 & $2(1,8)$ & $1(0,5)$ & $1(0,5)$ \\
\hline La Rioja & $1(0,7)$ & 0 & 0 & $1(0,8)$ & $2(1,1)$ & $2(1,9)$ & 0 & 0 & 0 & $1(0,5)$ \\
\hline Navarra & $2(1,5)$ & 0 & $1(0,5)$ & $1(0,8)$ & $11(6,3)$ & 0 & $3(1,1)$ & 0 & $4(2,2)$ & 0 \\
\hline País Vasco & $4(3,4)$ & 0 & 0 & $1(0,8)$ & $43(24,4)$ & $1(0,9)$ & $2(0,7)$ & 0 & $10(5,5)$ & $5(2,6)$ \\
\hline Región de Murcia & $6(3)$ & $4(5,4)$ & $1(0,5)$ & $7(5,3)$ & $2(1,1)$ & $2(1,9)$ & $5(1,8)$ & $3(2,6)$ & $8(4,4)$ & $5(2,6)$ \\
\hline Total de artículos & 134 & 74 & 211 & 132 & 176 & 106 & 274 & 114 & 182 & 191 \\
\hline
\end{tabular}

Tab. 8. Comparación entre revistas del número y porcentaje (entre paréntesis) de artículos sobre las comunidades autónomas en el periodo 2010-2018). El porcentaje está calculado por el número de artículos en cada caso.

nuación están Zephyrus (10), Saguntum (6), AEspA (4), Complutum (4), Munibe (2) y Pyrenae (2), faltando en $A P L$ y Lucentum.

En todas las revistas comparadas los porcentajes de estudios sobre otros territorios extrapeninsulares son similares: entre el 7 \% y el $10 \%$. Complutum y Saguntum son las excepciones. La primera tiene un $22,9 \%$ de artículos entre los que sobresalen los dedicados al continente africano. La explicación es la edición de un número monográfico ocasional en 2014 con 14 artículos sobre arte y paisaje en el Alto Atlas. En Saguntum, el $18,7 \%$ de trabajos sobre otras culturas mediterráneas de la Edad del Bronce se suelen referir a la cultura nurágica. 


\section{ANÁLISIS DE LA AUTORÍA}

\subsection{Análisis de la autoría en $T P$}

Los 933 documentos recogidos por ÍnDICEs CSIC en los 75 volúmenes de $T P$ se deben a 1219 autores diferentes. Los investigadores con más trabajos firmados se corresponden a las instituciones más ligadas con la trayectoria de la revista ${ }^{8}$ :

- Martín Almagro Basch (24 contribuciones), fundador de la revista, director del IEP del CSIC, del MAN y catedrático de la UCM (Mederos Martín 2017).

- Ignacio Montero Ruiz (20), MdA, IUOG e IH del CSIC.

- Martín Almagro Gorbea (19), UCM.

- Pilar López García (17), IEP e IH del CSIC.

- Manuel Fernández-Miranda Fernández (15), UCM.

- José Antonio López Sáez (14), UCM e IH del CSIC.

- Ramón Fábregas Valcarce (13), UNED, UVigo y USC.

- Teresa Chapa Brunet (13), UCM.

- Rodrigo de Balbín Behrmann (13), UCM y UAH.

- María Isabel Martínez Navarrete (12), UCM e IH del CSIC.

En cambio, se diversifica más la adscripción institucional de los autores más repetidos en los trabajos del periodo 2010-2018: Carlos P. Odriozola (6), de la Universidad de Sevilla, y Juan Francisco Gibaja Bao (5), que ha firmado como Institució Milà i Fontanals del CSIC, Museu d'Arqueologia de Catalunya y Universidade do Algarve. Este dato puede interpretarse como reflejo del cambio de estrategia editorial que se ha producido en la mayor parte de las publicaciones. Si estas nacieron en general con el objetivo prioritario de difundir las investigaciones de la institución editora, con el tiempo han modificado sus hábitos, incluyendo cada vez más contribuciones externas y procurando evitar la repetición continua de algunos autores. Las instituciones editoras, como las universidades o el propio CSIC, han desarrollado en los últimos años políticas de mayor control sobre las revistas para cumplir estándares de excelencia científica (criterios Latindex, de las agencias de evaluación y de selección en diferentes bases de datos e índices de citas). Aunque se han reducido presupuestos, la edición en Internet y el reconocimiento internacional promueve que las mejores revistas reciban más originales, potenciando la diversidad en la autoría.

El grado de coautoría de los artículos es un indicador del trabajo en equipo. Esta circunstancia puede

\footnotetext{
${ }^{8}$ Véase lista de abreviaturas al final del artículo.
}

deberse a la formación de grupos de investigación o en ocasiones a la colaboración interdisciplinar relacionada con los cambios teórico-metodológicos en la disciplina. En el total de 933 registros de TP en ÍnDICEs CSIC figuran 2171 firmas, con una media de 2,3 por documento. En 475 (51 \%) solo hay un único autor firmante, mientras que encontramos 1 anónimo, 184 trabajos con dos autores, 99 de tres, 60 de cuatro y 114 con cinco o más. El primer artículo con más de 10 firmantes se editó en 2002, apareciendo artículos con 15 en 2006 y 2015. La tabla 9 muestra una tendencia clara al incremento en el número de autores por documento y la progresiva reducción del porcentaje de trabajos con una única firma.

Respecto a la distribución por género en $T P$, han participado 420 mujeres como autoras, un $34 \%$ del total de diferentes autores. En la trayectoria de la revista este porcentaje se ha mantenido sin grandes variaciones, entre el $30 \%$ en el periodo inicial (19601979) y un $38 \%$ en 2000-2009, descendiendo a un $33 \%$ en la última década.

Otro aspecto de interés es la filiación institucional de los autores. El CSIC, como entidad editora, tiene el mayor número de contribuciones (192), si bien en esta cifra se engloban aportaciones de sus diferentes centros repartidos por varias comunidades autónomas y en Roma. Tras el CSIC figura la UCM (168), UAM (44), UAB (39), USC (34), UGR (31), MAN (27), UC (27), URV (26), US (26), UNED (25), UB (25), UV (22) y UAH (21). En total hay autores de 43 universidades españolas diferentes, así como museos e instituciones de investigación, situados en todas las comunidades autónomas.

Si se analiza la evolución de las instituciones más frecuentes en la filiación de los autores (Tab. 10) se aprecia que la UCM ocupó el primer lugar en las primeras décadas de la revista, pero ha ido perdiendo presencia a medida que se reforzaba su propia revista Complutum. El CSIC como entidad editora ha mantenido una aportación constante por debajo del $25 \%$ de los estudios.

\begin{tabular}{|c|c|c|c|}
\hline Periodo & Documentos & $\begin{array}{c}\text { Media de } \\
\text { autores por } \\
\text { documento }\end{array}$ & $\begin{array}{c}\text { Documentos } \\
\text { con un único } \\
\text { autor \% }\end{array}$ \\
\hline $1960-79$ & 198 & 1,2 & 81 \\
\hline $1980-89$ & 143 & 1,5 & 70 \\
\hline $1990-99$ & 203 & 2,1 & 49 \\
\hline $2000-09$ & 198 & 2,8 & 39 \\
\hline $2010-18$ & 191 & 3,9 & 19 \\
\hline
\end{tabular}

Tab. 9. Evolución de la media de autores por documento y del porcentaje de trabajos con un único autor firmante en Trabajos de Prehistoria entre 1960 y 2018. 


\begin{tabular}{|c|c|c|c|}
\hline Periodo & Documentos & $\begin{array}{c}\text { Filiaciones más } \\
\text { frecuentes en los } \\
\text { autores }\end{array}$ & $\begin{array}{c}\text { Porcentaje } \\
\text { editor \% }\end{array}$ \\
\hline $1960-79$ & 198 & $\begin{array}{c}\text { UCM (58), CSIC } \\
(36), \text { MAN (13) }\end{array}$ & 18 \\
\hline $1980-89$ & 143 & $\begin{array}{c}\text { UCM (38), CSIC } \\
(28), \text { UAM (10) }\end{array}$ & 20 \\
\hline $1990-99$ & 203 & $\begin{array}{c}\text { CSIC (45), UCM } \\
(37), \text { USC (17) }\end{array}$ & 22 \\
\hline $2000-09$ & 198 & $\begin{array}{c}\text { CSIC (39), UCM } \\
(19), \text { URV (13) }\end{array}$ & 20 \\
\hline $2010-18$ & 191 & $\begin{array}{c}\text { CSIC (44), UAB } \\
(20), \text { UCM y } \\
\text { UGR (16) }\end{array}$ & 23 \\
\hline
\end{tabular}

Tab. 10. Evolución de las instituciones más frecuentes en la filiación de los autores y del porcentaje de la entidad editora en TP entre 1960 y 2018.

Pese a que puede concluirse que en los últimos años ha aumentado ligeramente el número de trabajos firmados por investigadores del CSIC, también debe tenerse en cuenta que cada vez más se trata de trabajos en colaboración con autores de otras instituciones (Tab. 11).

\begin{tabular}{|c|c|c|c|}
\hline Periodo & $\begin{array}{c}\text { Documentos } \\
\text { con autores } \\
\text { CSIC }\end{array}$ & $\begin{array}{c}\text { Documentos } \\
\text { con autores } \\
\text { CSIC y otras } \\
\text { filiaciones }\end{array}$ & $\begin{array}{c}\text { Porcentaje } \\
\text { en } \\
\text { colaboración } \\
\text { \% }\end{array}$ \\
\hline $1960-79$ & 36 & 4 & 11 \\
\hline $1980-89$ & 28 & 5 & 18 \\
\hline $1990-99$ & 45 & 26 & 58 \\
\hline $2000-09$ & 39 & 23 & 59 \\
\hline $2010-18$ & 44 & 37 & 84 \\
\hline
\end{tabular}

Tab. 11. Evolución de artículos de investigadores del CSIC y porcentaje de trabajos en colaboración con autores de otras instituciones, en Trabajos de Prehistoria entre 1960 y 2018.

\subsection{Análisis de la autoría en comparación con otras revistas}

La tabla 12 muestra la situación actual respecto a la procedencia de los autores con mayor número de artículos en las diez revistas seleccionadas en este estudio. En 5 casos el autor más frecuente es ajeno a la entidad editora ${ }^{9}$ y en 3 su aportación supone menos de un artículo cada 2 años.

\footnotetext{
${ }^{9}$ El significado de las abreviaturas de las instituciones editoras o de filiación de los autores se especifica en un listado al final del artículo.
}

\begin{tabular}{|l|c|}
\hline $\begin{array}{l}\text { Revista y entidad } \\
\text { editora }\end{array}$ & $\begin{array}{c}\text { Autor más frecuente 2010-2018 y } \\
\text { filiación }\end{array}$ \\
\hline AEspA (CSIC) & $\begin{array}{c}\text { Margarita Orfila Pons (UGR), Antonio } \\
\text { Pizzo (CSIC) y Javier Salido } \\
\text { Domínguez (CSIC) }=3 \text { trabajos }\end{array}$ \\
\hline APL (SIP) & Ferran Arasa i Gil (UV) $=5$ \\
\hline $\begin{array}{l}\text { Complutum } \\
\text { (UCM) }\end{array}$ & Jorge de Torres Rodríguez (UCM) $=6$ \\
\hline Lucentum (UA) & Rosario Cebrián Fernández (UCM) $=5$ \\
\hline Munibe A-A (SCA) & $\begin{array}{c}\text { Francisco Etxeberría Gabilondo (UPV) } \\
=8\end{array}$ \\
\hline Pyrenae (UB) & $\begin{array}{c}\text { Francisco Gracia Alonso (UB) y Jordi } \\
\text { Vidal Palomino (UAB) }=3\end{array}$ \\
\hline Saguntum (UV) & Ferran Arasa i Gil (UV) $=12$ \\
\hline Spal (US) & $\begin{array}{c}\text { Enrique García Vargas (US) y Alfredo } \\
\text { Mederos Martín (UAM) }=4\end{array}$ \\
\hline TP (CSIC) & Carlos P. Odriozola (US) $=6$ \\
\hline Zephyrus (USAL) & Diego Gárate Maidagán (AM) $=6$ \\
\hline
\end{tabular}

Tab. 12. Autores más frecuentes por revista en el periodo 2010-2018.

El incremento de la coautoría en $T P$ destaca en la comparación por revistas desde 2010 (Tab. 13). Esto puede deberse a la mayor pervivencia de los hábitos de trabajo tradicionales en los estudios de Historia antigua (con mayor presencia en algunas de las publicaciones consideradas).

\begin{tabular}{|l|c|c|c|}
\hline Revista & Documentos & $\begin{array}{c}\text { Media de } \\
\text { autores por } \\
\text { documento }\end{array}$ & $\begin{array}{c}\text { Documentos } \\
\text { de un autor } \\
\text { \% }\end{array}$ \\
\hline AEspA & 133 & 2,1 & 46 \\
\hline APL & 74 & 2,4 & 42 \\
\hline Complutum & 211 & 1,9 & 64 \\
\hline Lucentum & 132 & 1,7 & 54 \\
\hline Munibe $A-A$ & 176 & 3,5 & 30 \\
\hline Pyrenae & 106 & 1,8 & 53 \\
\hline Saguntum & 274 & 2,7 & 40 \\
\hline Spal & 114 & 2,2 & 45 \\
\hline TP & 191 & 3,9 & 19 \\
\hline Zephyrus & 182 & 2,8 & 31 \\
\hline
\end{tabular}

Tab. 13. Media de autores por documento y del porcentaje de trabajos de un único autor entre 2010 y 2018 en las revistas comparadas.

La comparación sobre las instituciones con mayor contribución en cada revista (Tab. 14) muestra, en ge-

Trab. Prehist., 76, N. ${ }^{\circ}$ 2, julio-diciembre 2019, pp. 199-218, ISSN: 0082-5638

https://doi.org/10.3989/tp.2019.12233 
neral, aportaciones de los autores de la entidad editora inferiores al $25 \%$. Los casos extremos son Saguntum por arriba y Zephyrus por abajo.

\begin{tabular}{|c|c|c|c|}
\hline Revista & Documentos & $\begin{array}{l}\text { Filiaciones más } \\
\text { frecuentes en los } \\
\text { autores }\end{array}$ & $\begin{array}{l}\text { Porcentaje } \\
\text { editor } \%\end{array}$ \\
\hline$A E s p A$ & 133 & $\begin{array}{l}\text { CSIC (16), US } \\
\text { (13), UCM (12) }\end{array}$ & 12 \\
\hline$A P L$ & 74 & $\begin{array}{c}\text { UV (30), SIP (12), } \\
\text { UA (7) }\end{array}$ & 16 \\
\hline Complutum & 211 & $\begin{array}{c}\text { UCM (39), CSIC } \\
\text { (12), UB y UGR } \\
\text { (8) }\end{array}$ & 18 \\
\hline Lucentum & 132 & $\begin{array}{c}\text { UA (16), UV (11), } \\
\text { UGR (10) }\end{array}$ & 12 \\
\hline Munibe $A-A$ & 176 & $\begin{array}{l}\text { UPV (44), SCA } \\
(22), \text { UCM (16) }\end{array}$ & 13 \\
\hline Pyrenae & 106 & $\begin{array}{c}\text { UB (22), UAB } \\
\text { (13), UNIZAR (5) }\end{array}$ & 21 \\
\hline Saguntum & 257 & $\begin{array}{c}\text { UV (84), SIP (19), } \\
\text { UA (18) }\end{array}$ & 33 \\
\hline Spal & 114 & $\begin{array}{c}\text { US (29), UGR y } \\
\text { UAM (8) }\end{array}$ & 25 \\
\hline$T P$ & 191 & $\begin{array}{c}\text { CSIC (44), UAB } \\
\text { (20), UCM y } \\
\text { UGR (16) }\end{array}$ & 23 \\
\hline Zephyrus & 182 & $\begin{array}{l}\text { CSIC (21), UCM, } \\
\text { UNED y UGR } \\
(13), \text { USAL (11) }\end{array}$ & 6 \\
\hline
\end{tabular}

Tab. 14. Comparación entre revistas de las instituciones más frecuentes en la filiación y porcentaje de la entidad editora.

La comparación de la lista de autores firmantes en cada revista en el periodo 2010-2018 (Tab. 15) muestra las coincidencias existentes. En todas hay más de un $10 \%$ de autores que también publicaron en $T P$. Destaca en número y porcentaje Zephyrus, con 108 autores en común (un $25 \%$ de los 427 que publicaron en Zephyrus y un $19 \%$ de los 567 autores diferentes que publicaron en $T P$ ).

\section{ANÁLISIS DE LA INTERNACIONALIDAD EN AUTORÍA Y LENGUA DE PUBLICACIÓN}

\subsection{Análisis de la internacionalidad en TP}

La participación extranjera en la autoría es un indicador del grado de internacionalidad de las revistas.

\begin{tabular}{|l|c|c|c|}
\hline Revista & $\begin{array}{c}\text { Total de } \\
\text { autores }\end{array}$ & $\begin{array}{c}\text { Coincidencia } \\
\text { con } \boldsymbol{T P}\end{array}$ & $\%$ \\
\hline AEspA & 245 & 32 & 13 \\
\hline APL & 139 & 30 & 22 \\
\hline Complutum & 342 & 70 & 20 \\
\hline Lucentum & 187 & 21 & 11 \\
\hline Munibe A-A & 438 & 84 & 19 \\
\hline Pyrenae & 175 & 23 & 13 \\
\hline Saguntum & 486 & 68 & 14 \\
\hline Spal & 209 & 39 & 19 \\
\hline Zephyrus & 427 & 108 & 25 \\
\hline
\end{tabular}

Tab. 15. Coincidencia entre los autores de Trabajos de Prehistoria y las otras 9 revistas entre 2010 y 2018.

En la trayectoria completa de TP hay 202 trabajos de autores extranjeros, individuales o en coautoría con investigadores españoles. Proceden principalmente de Francia (38 documentos), Reino Unido (35), Estados Unidos (32), Alemania (30), Portugal (28), Argentina e Italia (10). Esta participación, cuyo punto más bajo estuvo en la década de 1980, ha aumentado por encima del $30 \%$ en los últimos años, especialmente con contribuciones desde Portugal (Tab. 16).

\begin{tabular}{|l|c|c|c|c|}
\hline Periodo & Documentos & $\begin{array}{c}\text { Documentos } \\
\text { de autores } \\
\text { extranjeros }\end{array}$ & $\begin{array}{c}\text { Porcentaje } \\
\%\end{array}$ & $\begin{array}{c}\text { País de } \\
\text { procedencia } \\
\text { principal }\end{array}$ \\
\hline $\begin{array}{l}1960- \\
1979\end{array}$ & 198 & 39 & 20 & EE. UU. (8) \\
\hline $\begin{array}{l}1980- \\
1989\end{array}$ & 143 & 10 & 7 & EE. UU. (3) \\
\hline $\begin{array}{l}1990- \\
1999\end{array}$ & 203 & 45 & 22 & $\begin{array}{c}\text { Reino Unido } \\
(10)\end{array}$ \\
\hline $\begin{array}{l}2000- \\
2009\end{array}$ & 198 & 35 & 18 & EE. UU. (9) \\
\hline $\begin{array}{l}2010- \\
2018\end{array}$ & 191 & 70 & 37 & Portugal (21) \\
\hline
\end{tabular}

Tab. 16. Evolución de la participación de autores extranjeros en Trabajos de Prehistoria entre 1960 y 2018.

Entre 1960 y $2018 T P$ ha publicado 59 contribuciones en lenguas extranjeras (49 en inglés, 9 en francés y 1 en italiano). De forma creciente, pero aún escasa, algunos de estos artículos en inglés son de investigadores españoles que lo emplean para aumentar su difusión internacional. Así, de los 49 documentos en inglés, 30 se deben a autores extranjeros, 10 a 
investigadores ligados a instituciones españolas y en 9 colaboran españoles y colegas de otros países.

Otro indicador de internacionalidad es la composición de los Consejos de Redacción y Asesor ${ }^{10}$. TP ha contado con la contribución de vocales extranjeros en ambos, e incluso en la figura del director de la revista: Antonio Gilman en el reciente periodo (Díaz-delRío 2019).

\subsection{ANÁLISIS DE LA \\ INTERNACIONALIDAD EN COMPARACIÓN CON OTRAS REVISTAS}

En la comparativa con otras revistas para el periodo 2010-2018 (Tab. 17), solo Complutum alcanza un grado de internacionalidad similar a TP. Francia, Portugal y Reino Unido son los países de participación más frecuente.

\begin{tabular}{|l|c|c|c|c|}
\hline Revista & Artículos & $\begin{array}{c}\text { Artículos con } \\
\text { autores } \\
\text { extranjeros }\end{array}$ & $\begin{array}{c}\text { Porcentaje } \\
\%\end{array}$ & $\begin{array}{c}\text { País de } \\
\text { procedencia } \\
\text { principal }\end{array}$ \\
\hline AEspA & 133 & 24 & 18 & Francia (9) \\
\hline APL & 74 & 4 & 5 & EE. UU. (2) \\
\hline Complutum & 211 & 69 & 33 & $\begin{array}{c}\text { Reino Unido } \\
(18)\end{array}$ \\
\hline Lucentum & 132 & 12 & 9 & Alemania (6) \\
\hline $\begin{array}{l}\text { Munibe } \\
\text { A-A }\end{array}$ & 176 & 28 & 16 & $\begin{array}{c}\text { Reino Unido } \\
(9)\end{array}$ \\
\hline Pyrenae & 106 & 21 & 20 & Portugal (5) \\
\hline Saguntum & 257 & 61 & 22 & Francia (16) \\
\hline Spal & 114 & 19 & 17 & Portugal (15) \\
\hline TP & 191 & 70 & 37 & Portugal (21) \\
\hline Zephyrus & 182 & 29 & 16 & Francia (12) \\
\hline
\end{tabular}

Tab. 17. Comparación entre revistas de la participación de autores extranjeros entre 2010 y 2018.

Las normas de presentación de originales de las revistas dan la pauta de las lenguas admisibles para difundir la investigación y, por tanto, de la opción escogida por los autores (Tab. 2). En el periodo 20102018 (Tab. 18) Pyrenae, Complutum y Saguntum destacan por su mayor porcentaje de trabajos en inglés. Sin embargo en otras de las revistas comparadas les superan los artículos en otras lenguas. Esto puede atri-

${ }^{10}$ En Anexo 1 se adjunta la relación de miembros de estos órganos editoriales. buirse a su contenido (AEspA, Lucentum y Spal) o a la opción por la lengua vernácula de los autores $(A P L$, Pyrenae, Saguntum). Estos idiomas no deben interpretarse al mismo nivel en el esfuerzo de internacionalización de las revistas.

\begin{tabular}{|c|c|c|c|c|}
\hline Revista & Artículos & $\begin{array}{l}\text { Artículos } \\
\text { en inglés }\end{array}$ & $\begin{array}{c}\text { Porcentaje } \\
\%\end{array}$ & Otras lenguas \\
\hline$A E s p A$ & 135 & 2 & 1 & $\begin{array}{c}7 \text { (3 portugués, } \\
2 \text { francés, } 2 \\
\text { italiano) }\end{array}$ \\
\hline$A P L$ & 74 & 0 & 0 & 8 (catalán) \\
\hline Complutum & 211 & 55 & 26 & $\begin{array}{c}4 \text { (3 francés, } 1 \\
\text { portugués) }\end{array}$ \\
\hline Lucentum & 132 & 1 & 1 & $\begin{array}{c}7 \text { (3 italiano, } 2 \\
\text { francés, } 1 \\
\text { alemán, } 1 \\
\text { catalán) }\end{array}$ \\
\hline $\begin{array}{l}\text { Munibe } \\
A-A\end{array}$ & 176 & 17 & 10 & $\begin{array}{c}4 \text { (3 francés, } 1 \\
\text { euskera) }\end{array}$ \\
\hline Pyrenae & 106 & 47 & 44 & $\begin{array}{c}30 \text { (21 catalán, } \\
6 \text { francés, } 3 \\
\text { italiano) }\end{array}$ \\
\hline Saguntum & 274 & 52 & 19 & $\begin{array}{c}49 \text { (36 catalán, } \\
6 \text { italiano, } 5 \\
\text { francés, } 2 \\
\text { portugués) }\end{array}$ \\
\hline Spal & 114 & 5 & 4 & $\begin{array}{l}14 \text { (11 } \\
\text { portugués, } 2 \\
\text { francés, } 1 \\
\text { italiano) }\end{array}$ \\
\hline$T P$ & 191 & 36 & 19 & 2 (francés) \\
\hline Zephyrus & 182 & 20 & 11 & $\begin{array}{c}4 \text { (2 francés, } 1 \\
\text { italiano, } 1 \\
\text { portugués) }\end{array}$ \\
\hline
\end{tabular}

Tab. 18. Comparación entre revistas sobre la edición en inglés o en otras lenguas entre 2010 y 2018.

\section{ANÁLISIS DE DATOS DE CITACIÓN}

\subsection{Análisis de citación en $T P$}

$T P$ está recogido actualmente por los dos principales índices de citas internacionales, Web of Science y Scopus. En WoS aparece en la base Arts \& Humanities Citation Index (categoría Archaeology, carente de indicadores bibliométricos) y en Social Science Citation Index (categoría Anthropology). Gracias a su pertenencia a este segundo fichero está incluida en los informes anuales, conocidos como Journal Citation Reports $(J C R)$, donde se ofrece el factor de impacto (FI). Este

Trab. Prehist., 76, N. ${ }^{\circ}$ 2, julio-diciembre 2019, pp. 199-218, ISSN: 0082-5638

https://doi.org/10.3989/tp.2019.12233 
se calcula por las citas recibidas en un año a los contenidos de los dos inmediatamente anteriores. Es decir, este tipo de baremo obvia que la obsolescencia en estas disciplinas no es comparable con la de las ciencias experimentales. En JCR la revista estuvo clasificada en la categoría de Historia en los informes de 2009 a 2012, situándose en el segundo cuartil en tres de esos años, y en Antropología desde 2013, apareciendo en el tercer cuartil entre 2013 y 2016.

La inclusión en este índice es un mérito que avala la trayectoria de esta revista. Sin embargo, limitaremos nuestro siguiente análisis al producto competidor, $\mathrm{Sco}$ $p u s$, dado que ofrece una mayor cobertura de $T P$, así como de otros títulos que se comparan en este estudio (Tab. 2). Frente al intervalo de dos años aplicado en el ranquin tradicional por factor de impacto en $W o S$, los indicadores que se extraen de los datos de Scopus se establecen sobre 3 años de citación. Ello sigue siendo bastante insuficiente para valorar adecuadamente disciplinas cuyas publicaciones tienen escasa obsolescencia. El indicador CiteScore del propio productor de Scopus calcula el promedio de citas en un año a los artículos aparecidos en los tres inmediatamente anteriores. $T P$ figura en estas tablas con datos desde 2011 en la categoría Arqueología con un doble cálculo: para Humanidades y para Ciencias Sociales (con pocas diferencias entre ambos conjuntos). Como cada año se incorporan nuevos títulos, la posición en un ranquin depende de la proyección de los artículos y, además, de la composición general del conjunto, mejorando la posición en el ranquin en la medida en que se introduzcan revistas de temática similar o disminuyendo si los nuevos títulos se centran en otros ámbitos geográficos. A pesar de ello, hay cierta estabilidad y TP se ha mantenido generalmente en una media entre 0,52 y 0,56 citas por documento. Por ello suele estar en el segundo cuartil y a veces en los últimos títulos del primer cuartil. Su mejor resultado fue en 2013, cuando una media de 0,77 le situaba más claramente en el primer cuartil.

Además, la revista figura en los portales bibliométricos que utilizan datos de Scopus: Scimago Journal \& Country Rank (https://www.scimagojr.com/, del grupo de investigación Scimago) y CWTS Journals Indicators (http://www.journalindicators.com/, de la Universidad de Leiden). Ambos generan indicadores de citas para un intervalo de tres años, pero con un cálculo ponderado. Scimago Journal Rank (SJR), que considera la posición relativa de las revistas citantes, otorga una mejora para $T P$. Esto la sitúa en el primer cuartil entre 2004 y 2006 y entre 2012 y 2017 tanto en la categoría de Arqueología en Humanidades como en Ciencias Sociales, y en 2009 solo en Humanidades. Por el contrario, según el indicador Source Normalized Impact per Paper (SNIP) de la Universidad de Leiden (ponderado según los hábitos de citación de cada disciplina), queda solamente en el tercer cuartil en 2017.

Una búsqueda directa en Scopus proporciona datos de citación para el periodo 2003-2018 en TP. En disciplinas donde las citas suelen tener un largo recorrido solo una cobertura amplia muestra una imagen más real del impacto de los artículos. En febrero de 2019 Scopus reunía un total de 340 registros de $T P$, que habían recibido hasta ese momento un total de 1853 citas, con un índice h de 17. Este último indicador es una alternativa que se basa en los documentos más citados sin hacer una media por los artículos, por lo que aporta otro punto de vista a la hora de valorar las citas recibidas por autores o revistas. La búsqueda global ha recuperado los 30 documentos más citados (Anexo 2). Se añade el cálculo del promedio de citas recibidas por año para destacar los artículos cuyo impacto es proporcionalmente mayor. Esta lista muestra que no hay una vía única para alcanzar una alta citación. En ella hay artículos con una alta coautoría junto a otros de autor único, redactados en inglés o en español, y con diferentes enfoques temáticos y metodológicos.

A partir de esta misma búsqueda, se ha analizado la procedencia de las citas recibidas. Destaca Quaternary International (160 artículos con citas a TP), junto a la propia TP (141), seguidas de Journal of Archaeological Science (61), Journal of Archaeological Science Reports (48), Zephyrus (46), Complutum (44), Munibe Antropologia-Arkeologia (28), Archaeological and Anthropological Sciences (25), Quaternary Science Reviews (23) y Journal of Human Evolution (20).

Es relevante que 6 de estos 10 títulos son internacionales y publican en inglés, pero en parte puede tratarse de artículos de investigadores españoles. Si se analiza la filiación de los autores citantes las instituciones de mayor frecuencia son la Universitat Rovira i Virgili (presente en 131 documentos citantes), CSIC (129), Institut Català de Paleoecologia Humana i Evolució Social (IPHES, 128), Universitat Autònoma de Barcelona (100), Universidad Complutense de Madrid (88), Universitat de Barcelona (83) y Universidad Autónoma de Madrid (79), para llegar a la primera no nacional: el Centre national de la recherche scientifque $(C N R S)$ con 77 trabajos.

La base de datos Scopus nos permite también conocer el país de procedencia de los autores citantes. España ocupa lógicamente el primer lugar con 924 documentos, seguida con frecuencias notables por Reino Unido (180), Francia (141), Estados Unidos (104), Alemania (88), Portugal (84), Italia (57), Australia (26) y China (26). Sin duda, la creciente cooperación científica internacional en la investigación española está contribuyendo en la apreciable difusión y visibilidad de TP. 


\subsection{Análisis de citación en comparación con otras revistas}

$T P$ fue la primera revista española de Arqueología y Prehistoria incorporada en los índices de citas, sin duda gracias a los buenos resultados obtenidos en los estudios realizados por los grupos de investigación especializados en bibliometría (Martínez Navarrete y Montero Ruiz 2016). En Scopus presenta datos desde 2003, anticipándose a los restantes títulos comparados en este estudio: AEspA (desde 2009); APL (2010); Complutum y Zephyrus (2011); Munibe. AntropologiaArkeologia (2013); Lucentum, Pyrenae y Spal (2015) y finalmente Saguntum (2017).

La situación actual distancia con claridad a $T P$ de las revistas españolas de su especialidad en número de citas recibidas (Fig. 3). En los primeros años fue creciendo a pesar de ser el único título español de la especialidad, pero sus números se han incrementado notablemente al enriquecerse las revistas fuente. Según la búsqueda en Scopus a 3 de febrero de 2019, TP había recibido 323 citas en 2018, a gran distancia de Zephyrus (89), Complutum (49), AEspA (45), Munibe Antropologia-Arkeologia (33), APL (18), SPAL (16),

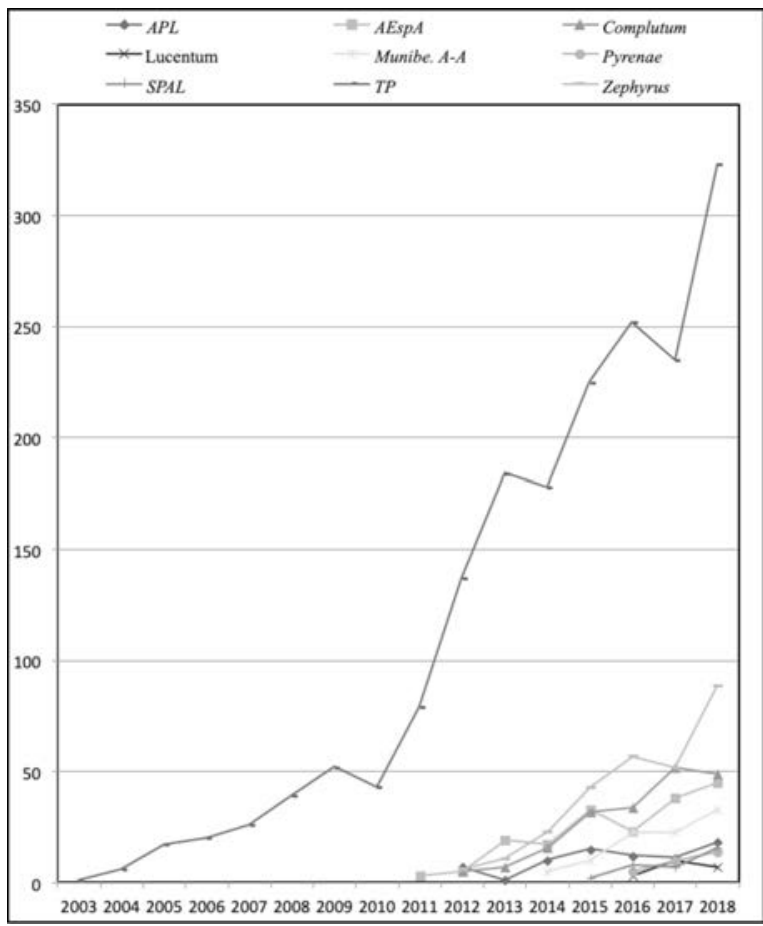

Fig. 3. Citas recibidas por las revistas comparadas de acuerdo a los datos recogidos en Scopus. Búsqueda 3/2/2019.
Pyrenae (14), Lucentum (7) y Saguntum (0) ${ }^{11}$. Estos datos recogen las citas recibidas al total de artículos de cada título en Scopus, en donde TP figura con bastante anticipación. La presencia creciente de títulos españoles de la especialidad contribuye a aumentar las citas recibidas por todas ellas, pero solamente Zephyrus mantiene una tendencia de crecimiento comparable con la experimentada por $T P$.

Los últimos indicadores bibliométricos publicados corresponden a las citas recibidas en 2018 a los años inmediatamente anteriores ${ }^{12}$, de modo que en este caso no influye la amplitud de la cobertura de cada título. En estos datos TP alcanza también una posición claramente destacada en los indicadores medidos a partir de Scopus respecto a las revistas españolas incluidas en este estudio (Tab. 19).

\begin{tabular}{|l|c|c|c|c|c|}
\hline Revista & $\begin{array}{c}\text { CiteScore } \\
\mathbf{2 0 1 8}\end{array}$ & $\begin{array}{c}\text { Percentil } \\
\text { Citescore }\end{array}$ & $\begin{array}{c}\text { SJR } \\
\mathbf{2 0 1 8}\end{array}$ & $\begin{array}{c}\text { Cuartil } \\
\text { SJR }\end{array}$ & SNIP \\
\hline AEspA & 0,22 & 47 & 0,132 & Q3 & 0,616 \\
\hline Complutum & 0,24 & 49 & 0,228 & Q2 & 0,357 \\
\hline Lucentum & 0,11 & 28 & 0,123 & Q3 & 0,427 \\
\hline Munibe A-A & 0,39 & 63 & 0,252 & Q2 & 0,291 \\
\hline Pyrenae & 0,26 & 52 & 0,245 & Q2 & 0,941 \\
\hline Spal & 0,39 & 63 & 0,288 & Q2 & 0,648 \\
\hline TP & 0,74 & 79 & 0,511 & Q1 & 0,973 \\
\hline Zephyrus & 0,62 & 75 & 0,418 & Q1 & 0,792 \\
\hline
\end{tabular}

Tab. 19. Comparación entre revistas de la posición en 2018 en diferentes indicadores elaborados a partir de los datos de Scopus. CiteScore (Elsevier) indica el número de citas recibidas en 2018 a los artículos de 2015-2017; el percentil indica la posición en el ranquin de Arqueología- Humanidades siendo 100 la primera; SJR Scimago Journal Rank y SNIP Source Normalized Impact per Paper (Universidad de Leiden) son cálculos ponderados a partir del valor de CiteScore. En el caso de $S J R$ se indica la posición en el cuartil en la categoría Arqueología-Humanidades siendo la mejor posición Q1 primer cuartil, seguido de Q2 segundo cuartil y Q3 tercer cuartil.

Para comparar con otras revistas los datos de búsquedas en Scopus se acotan las fechas al periodo 2010-

${ }^{11}$ La revista Saguntum se excluye de la figura 3 porque carecía de citas recibidas en el momento de la búsqueda: su cobertura se inicia en 2017.

12 Los indicadores de 2018 se basan en las citas recibidas en dicho año a los artículos publicados entre 2015 y 2017 . Por ello se excluye de la tabla 19 Saguntum cuya cobertura se inicia en 2017 y Archivo de Prehistoria Levantina sobre la que no se editaron datos bibliométricos en 2018. [Se realiza la comparación respecto a/La comparación se refiere a la posición en la categoría Arqueología en Humanidades, aunque algunos de estos títulos [pueden localizarse/ aparecen] también en otros epígrafes como Historia o Estudios Clásicos, con un ranquin distinto al compararse con diferentes conjuntos de revistas.

Trab. Prehist., 76, N. ${ }^{\circ}$ 2, julio-diciembre 2019, pp. 199-218, ISSN: 0082-5638

https://doi.org/10.3989/tp.2019.12233 
2018. Ello implica analizar un menor número de citas recibidas por $T P$ respecto a las del periodo completo 2003-2018, pero no reduce, sin embargo, su notable diferencia con las restantes publicaciones (Tab. 20). Se excluyen las revistas cuya cobertura en Scopus se inicia más tarde: Lucentum (2015), Munibe (2013), Pyrenae (2015), Saguntum (2017) o Spal (2015). Sí se incluyen Complutum y Zephyrus aunque en ambas los datos se recogen solo desde 2011.

\begin{tabular}{|l|c|c|c|c|}
\hline Revista & $\begin{array}{c}\text { Documentos } \\
\mathbf{2 0 1 0 - 2 0 1 8}\end{array}$ & $\begin{array}{c}\text { Índice } \\
\mathbf{h}\end{array}$ & $\begin{array}{c}\text { Citas } \\
\text { recibidas }\end{array}$ & $\begin{array}{c}\text { Media de } \\
\text { citas por } \\
\text { documento }\end{array}$ \\
\hline AEspA & 134 & 5 & 146 & 1,09 \\
\hline APL & 50 & 4 & 79 & 1,58 \\
\hline Complutum & 206 & 6 & 199 & 0,97 \\
\hline TP & 200 & 13 & 773 & 3,87 \\
\hline Zephyrus & 141 & 9 & 285 & 2,02 \\
\hline
\end{tabular}

Tab. 20. Comparación entre revistas de las citas recibidas a los artículos del periodo 2010-2018 recogidos en Scopus. Búsqueda $3 / 2 / 2019$.

En los datos de 2010-2018, TP se sitúa por delante de los restantes títulos comparados en cuanto al índice $\mathrm{h}$, que se basa en la distribución de las citas recibidas. El valor del índice indica que hay $\mathrm{h}$ artículos que recibieron h citas o más. Este cálculo favorece teóricamente a las revistas que más artículos publican. Sin embargo, $T P$ también destaca en la media de citas por documento.

\section{DISCUSIÓN Y CONCLUSIONES}

El estudio de la trayectoria completa de $T P$ desde su origen en 1960 nos ha permitido describir sus principales características y resaltar las tendencias de cambio. En comparación con el estudio aparecido al cumplirse los primeros 50 volúmenes (Rodríguez Alcalde et al. 1993), la revista se ha transformado en una publicación semestral con un creciente impacto nacional e internacional. De acuerdo con las directrices de Editorial CSIC, $T P$ ha adaptado sus características formales y de fondo para cumplir los criterios de selección de bases de datos y sistemas de evaluación. La labor del equipo editorial, con la contribución de expertos externos en la evaluación por pares, ha permitido mejorar los trabajos aprobados con el intercambio de sugerencias a los autores. Se ha reforzado su carácter especializado en Prehistoria y Protohistoria de la península ibérica, Baleares y Canarias, si bien se busca la proyección internacional con la promoción de textos en inglés, así como la participación de autores extranjeros.

Estas políticas editoriales favorecen la presencia en bases de datos e índices de citas, un indicador de reconocimiento que a su vez redunda en una mayor afluencia de nuevos textos. La categorización de $T P$ dentro del área de Antropología en $W o S$ le ha permitido entrar en el ranquin de revistas de $J C R$ que incluye Ciencias Sociales y no Humanidades, siendo por tanto la única revista española de Arqueología con factor de impacto. Al surgir el producto competidor Scopus, se ha incorporado en otros ránquines en los que progresivamente sí puede compararse con más publicaciones españolas. En estos productos, los títulos especializados en ámbitos nacionales, editados generalmente por organismos públicos sin ánimo de lucro, deben competir con productos editoriales de entidades multinacionales dirigidos a audiencias globales.

De acuerdo con la línea editorial de $T P$, la revista se centra en el I milenio a. C. En esta orientación coincide sobre todo con APL, Munibe y Zephyrus. TP puede considerarse la principal revista de referencia en España especialmente para los estudios centrados en el Calcolítico y la Edad del Bronce en el territorio peninsular, hecho que la diferencia respecto a otras revistas analizadas en este artículo. Este enfoque está acompañado desde el año 2000 de un refuerzo significativo de contribuciones sobre Paleolítico y Neolítico, revitalizando una segunda línea de estudios menos atendida en las décadas de 1980 y 1990.

Las temáticas principales de estos períodos no han experimentado demasiados cambios en la trayectoria de $T P$, destacando tanto en el Calcolítico/Edad del Bronce como en el Paleolítico/Epipaleolítico los centrados en la cultura material (industrias lítica, ósea, metalúrgica y cerámica) y sus tipologías. Por otra parte, el arte rupestre es mayoritario en aquellas contribuciones centradas en la Prehistoria antigua y en la Edad del Bronce, lo cual es un rasgo característico de las culturas de estos periodos. Los relativos a enterramientos y ritos funerarios son igualmente importantes en la Edad del Bronce. Los estudios sobre metodología y patrimonio arqueológicos son menos frecuentes en $T P$ desde el año 2000, en contraste con Complutum, que podría ser para estos casos la revista de referencia en España.

La distribución territorial de los estudios está más equilibrada en las revistas editadas en Madrid que en los restantes títulos que enfatizan más su propia comunidad. El análisis espacial en TP muestra su tendencia a abarcar todo el territorio peninsular. Destacan en especial la Región Cantábrica, el Sureste, Baleares $\mathrm{y}$, en la última década, Cataluña.

Las revistas académicas cumplen un servicio fundamental para el desarrollo de las disciplinas científi- 
cas y la evaluación de la actividad investigadora en España. Muchas se fundaron para difundir las investigaciones de la institución editora, pero progresivamente se han adaptado a las exigencias de profesionalización e internacionalización, adoptando criterios cada vez más estrictos en la selección de originales y dando servicio a una amplia comunidad científica. Un indicador de este salto de calidad es la generalización de la evaluación por pares externos.

Los indicadores bibliométricos han contribuido a que se centre la atención sobre la calidad de las revistas, incrementando en consecuencia la demanda de los autores por publicar en ellas. Numerosos investigadores han criticado públicamente "la tendencia excesiva a la cuantificación (al peso) y a la bibliometrización de la evaluación; [pues] no debería elevarse a los altares el factor de impacto de las revistas, sino la contribución al conocimiento" (Sanz 2019). Aunque compartimos estas opiniones, esto no debe conducir a desechar los estudios sobre la calidad de las publicaciones, que deben utilizarse en su justa medida.

En este panorama, $T P$ se ha consolidado como una revista abierta en la procedencia de los autores, con un elevado reconocimiento. En los últimos años se ha diversificado el número y procedencia de los autores. Aunque el CSIC como entidad editora se mantiene como la institución con mayor aportación de artículos, su peso no supera el $25 \%$. Este cómputo engloba aportaciones de diferentes centros repartidos por varias comunidades autónomas y en Roma. El porcentaje ideal de autores de la entidad editora se ha considerado cada vez de forma más exigente en los sistemas de evaluación de revistas. En el sistema Latindex se cifra en el $50 \%$, baremo que viene aplicándose desde la creación del catálogo en 2002. En España, la Comisión Nacional Evaluadora de la Actividad Investigadora (CNEAI) estableció un límite del $25 \%$ en 2002 como criterio para medir la endogamia editorial (Delgado et al. 2006: 17). Por su parte la FECYT ha considerado que el número de autores pertenecientes al consejo de redacción y la entidad editora no deben superar el $20 \%$, límite establecido en las convocatorias para obtener el sello de calidad editorial y científica de las revistas españolas que se vienen realizando desde 2007.

En su trayectoria, y en comparación con otros títulos, destaca el aumento de la coautoría, lo que incrementa el número total de autores y también caracteriza la participación actual de los investigadores del propio CSIC. Otro aspecto de interés es la tendencia a la internacionalización, que se muestra en un creciente porcentaje de autores extranjeros, que en el caso de $T P$ se manifiesta también en su participación en el Consejo de Redacción y en la dirección de la revista. También son indicadores de internacionalidad la edición de artículos en inglés y las citas procedentes de publicaciones no españolas. Sin duda, la edición electrónica en acceso abierto y la presencia en índices de citas ha contribuido notablemente a su difusión más allá de la península ibérica sobre la que se centra sus artículos.

En el estudio se han aprovechado los datos ofrecidos por Scopus, como índice de citas con mejor cobertura sobre revistas españolas de la disciplina. Este tipo de recursos internacionales aportan datos de interés para su evaluación, que no debe reducirse a los indicadores de citación. Son datos relativos para valorar la calidad pues miden solo parcialmente su impacto. La situación de una revista en un ranquin depende también de la presencia o no de publicaciones similares interesadas en los mismos temas dentro de la cobertura de los índices. Además, el que los estudios locales ocupen un lugar relevante en el área de conocimiento de Arqueología y Prehistoria no favorece, en principio, la visibilidad de sus resultados.

Subrayamos la notable incorporación de revistas españolas de la disciplina en los índices de citas que ya permite comparaciones entre ellas empleando estas bases de datos internacionales. El que su cobertura temporal siga siendo aún corta es un argumento adicional para limitar el alcance actual de los indicadores de citación en la evaluación de la excelencia científica. La temática común y la confluencia de un número de autores coincidentes con todos los títulos seleccionados muestran una comunidad de investigadores que comparten un conjunto de revistas. La comparación entre las revistas españolas de Humanidades y Ciencias Sociales presentes en Scopus o WoS sin duda podrá mejorarse en los próximos años, pues algunos títulos se han incorporado hace relativamente poco. TP obtiene unos resultados muy positivos en esta comparación. En los datos globales se beneficia de su mayor cobertura, pero también mantiene una posición destacada en los indicadores basados en los últimos 3 años. La comparación del periodo intermedio 2010-2018, la sitúa por delante de las restantes publicaciones españolas de la especialidad tanto en los indicadores que contabilizan las citas recibidas sólo por los trabajos más citados (índice h) como por media de citas por documento.

Como la cobertura de TP es ya suficientemente amplia en Scopus, este índice de citas permite estudios complementarios mediante búsquedas. Así, la lista de artículos más citados muestra la larga vigencia de la bibliografía científica en este campo, sin que pueda determinarse una única estrategia que favorezca la citación.

El análisis de citas confirma que la visibilidad internacional de las revistas españolas consideradas tiene que ver tanto con la lengua de publicación como con la coincidencia de autores entre revistas citantes y citadas. Se trata en realidad de dos factores combinados en los mismos casos: los autores españoles que

Trab. Prehist., 76, N. ${ }^{\circ}$ 2, julio-diciembre 2019, pp. 199-218, ISSN: 0082-5638

https://doi.org/10.3989/tp.2019.12233 
utilizan el inglés en revistas extranjeras citan trabajos en español, en ocasiones de su propia autoría. Igualmente, por la temática tratada (Prehistoria de la península ibérica), cabe esperar que las citas se incrementen si los índices incluyen más revistas españolas o publicaciones internacionales con participación frecuente de autores de España o Portugal.

El mayor porcentaje de coautoría en $T P$ frente a otros títulos puede contribuir a una mayor citación posterior, pero algunos artículos de un único autor han recibido también un elevado número de citas. Por ello, no se puede concluir que sea un factor determinante. $T P$ tiene otras características que pueden actuar como ventajas competitivas: la frecuencia semestral más ágil que los anuarios, la temprana incorporación de la evaluación externa por pares, la continuidad y regularidad de la edición garantizada por la estructura editorial del CSIC, así como la influencia y vinculación con el CINDOC que favoreció la normalización de la revista y cuyos estudios bibliométricos fueron elementos relevantes para que se incluyera en $W o S$ y Scopus.

El estudio bibliométrico de $T P$, en comparación con otros títulos de similar enfoque y difusión, permite una caracterización general de las pautas de difusión científica de los arqueólogos orientados hacia la Prehistoria y/o hacia la Historia antigua en la última década. Las iniciativas editoriales españolas en este ámbito son de carácter institucional con financiación muy limitada, pero deben competir en el marco internacional dominado por empresas multinacionales. Además, la posición en los indicadores bibliométricos extraídos de los índices de citas depende entre otros factores de la adecuación de las categorías temáticas y de la mayor o menor presencia en el mismo conjunto de otros títulos de perfil temático similar. Los excelentes resultados obtenidos por $T P$ en estos índices son reflejo del crecimiento de numerosos grupos de investigación españoles que han apostado por el trabajo en equipos multidisciplinares y la publicación en revistas nacionales e internacionales. El creciente grado de coautoría es una muestra de la transformación de la investigación arqueológica especializada en Prehistoria y Protohistoria frente a los hábitos tradicionales de la arqueología clásica que predominan en otros títulos. Todo ello tampoco sería posible sin la importante contribución altruista de evaluadores externos y consejos editoriales que conforman una comunidad científica comprometida con una difusión seria de la ciencia.

\section{ANEXOS: ARCHIVOS COMPLEMENTARIOS}

En la versión en línea (menú Herramientas del artículo) pueden consultarse dos archivos complementarios en formato Excel:
AC1 Relación de miembros del Consejo de Redacción y Consejo Asesor de Trabajos de Prehistoria.

AC2 Análisis de los 30 artículos más citados de Trabajos de Prehistoria según los datos recogidos en Scopus (3/2/2019).

\section{LISTA DE ABREVIATURAS}

$\begin{array}{ll}\text { AEspA } & \text { Archivo Español de Arqueología } \\ \text { AM } & \text { Arkelogi Museoa (Bilbao) } \\ \text { APL } & \text { Archivo de Prehistoria Levantina } \\ \text { CSIC } & \text { Consejo Superior de Investigaciones } \\ & \text { Científicas } \\ \text { IEP } & \text { Instituto Español de Prehistoria } \\ \text { IH } & \text { Instituto de Historia } \\ \text { IUOG } & \text { Instituto Universitario Ortega y Gasset } \\ \text { MAN } & \text { Museo Arqueológico Nacional } \\ \text { MdA } & \text { Museo de América } \\ \text { Munibe A-A } & \text { Munibe Antropologia-Arkeologia } \\ \text { RGZ } & \text { Römisch-Germanisches Zentralmuseum } \\ & \text { (Mainz) } \\ \text { SCA } & \text { Sociedad de Ciencias Aranzadi } \\ \text { SIP } & \text { Servicio de Investigación Prehistórica de } \\ & \text { Valencia } \\ \text { UA } & \text { Universidad de Alicante } \\ \text { UAB } & \text { Universitat Autònoma de Barcelona } \\ \text { UAH } & \text { Universidad de Alcalá } \\ \text { UAM } & \text { Universidad Autónoma de Madrid } \\ \text { UB } & \text { Universitat de Barcelona } \\ \text { UC } & \text { Universidad de Cantabria } \\ \text { UCM } & \text { Universidad Complutense de Madrid } \\ \text { UGR } & \text { Universidad de Granada } \\ \text { UNED } & \text { Universidad Nacional de Educación a } \\ \text { UNIZAR } & \text { Distancia } \\ \text { Universidad de Zaragoza } & \text { Universidad del País Vasco } \\ \text { URV } & \text { Universitat Rovira i Virgili } \\ \text { US } & \text { Universidad de Sevilla } \\ \text { USAL } & \text { Universidad de Salamanca } \\ \text { USC } & \text { Universidade de Santiago de Compostela } \\ \text { UV } & \text { Universidad de Valencia } \\ \text { UVigo } & \text { Universidad de Vigo } \\ & \end{array}$

\section{BIBLIOGRAFÍA}

Abadal, E. (ed.) 2017: Revistas cientificas. Situación actual y retos de futuro. Universitat de Barcelona. Barcelona.

Armada, X.-L. 2009: "Indicadores bibliométricos, visibilidad y calidad de revistas científicas: en torno a Revista d'Arqueologia de Ponent". Revista d'Arqueologia de Ponent 19: 7-28.

Armada, X.-L. 2016: "Explorando el panorama actual de las publicaciones periódicas de Arqueología: Revista d'Arqueologia de Ponent en contexto". Revista d'Arqueologia de Ponent 26: 295-310, https://doi.org/10.21001/rap.2016.26.19

Bouso, M.; Rafel, N. y Alonso, N. (eds.) 2016: "Les revistes científiques d'arqueologia a debat: present i futur - Archaeological Journals: debate on their present and future". Revista d'Arqueologia de Ponent 26: 261-324. 
Delgado López-Cózar, E.; Ruiz-Pérez, R. y Jiménez-Contreras, E. 2006: La edición de revistas cientificas. Directrices, criterios y modelos de evaluación. FECYT. Madrid.

https:/calidadrevistas.fecyt.es/sites/default/files/informes/EdicionRCE.pdf

Díaz-del-Río, P. 2019: "Editorial: Trabajos de Prehistoria, a hombros de gigantes". Trabajos de Prehistoria 76 (1): 7-8.

García del Toro, M. A.; García Avolio, J. L.; Juarez Pérez, M. y López Guerao, M. A. 1999: "Estudio bibliométrico de la revista Archivo Español de Arqueología: foro nacional de investigación arqueológica". En XXIV Congreso Nacional de Arqueología (Cartagena 1997) 5: 329-338. Murcia.

Gilman, A. 2018: "Editorial: 25 años de evaluación por pares en Trabajos de Prehistoria". Trabajos de Prehistoria 75 (1): 7-8. https://doi.org/10.3989/tp.2018.12201

Martínez Navarrete, M. I. y Montero Ruiz, I. 2016: "Trabajos de Prehistoria: su trayectoria como revista de impacto internacional". Revista d'Arqueologia de Ponent 26: 311-320. https://doi.org/10.21001/rap.2016.26.20

Mederos Martín, A. 2017: "Martín Almagro Basch, un balance de su trayectoria científica (1934-1984)". Cuadernos de Prehistoria y Arqueología 43: 251-289. http://doi.org/10.15366/cupauam2017.43.010

Mora, G. 2002: "Archivo Español de Arqueología. Notas para una historia de la revista". Archivo Español de Arqueología 75: 5-20. https://doi.org/ 10.3989/aespa.2002.v75.125

Rodríguez Alcalde, Á; San Millán Bujanda, M. J.; Sánchez Nistal, J. M.; Chapa Brunet, T.; Martínez Navarrete, M. I. y Ruiz Zapatero, G. 1993: "Análisis bibliométrico de Trabajos de Prehistoria: un chequeo a la prehistoria española de las tres últimas décadas". Trabajos de Prehistoria 50: 11-37. https://doi.org/10.3989/tp.1993.v50.i0.487
Rodríguez Alcalde, Á; Sánchez Nistal, J. M.; Martínez Navarrete, M. I. y San Millán Bujanda, M. J. 1996: "Análisis bibliométrico de las revistas españolas de Prehistoria y Arqueología en los últimos diez años". Trabajos de Prehistoria, 53 (1): 37-58. https://doi.org/10.3989/tp.1996.v53.i1.404

Rodríguez Alcalde, A. L.; Martínez Navarrete, M. I.; Sánchez Nistal, J. M. y San Millán Bujanda, M. J. 1997: "El análisis bibliométrico como aportación a la historiografía. Las citas en Prehistoria y Arqueología”. En G. Mora y M. Díaz-Andreu (eds.): La cristalización del pasado: Génesis y desarrollo del marco institucional de la Arqueología en España. Universidad de Málaga. Málaga: 695-704.

Román Román, Adelaida 2003: Índices de impacto de las revistas españolas de Antropología, Arqueología y Prehistoria, Historia de América, Lengua Española y Filologías Griega y Latina. Informe. http://eprints.rclis.org/6020/

Román Román, A. y Alcain Partearroyo, M. D. 2005: "Las revistas españolas de Prehistoria y Arqueología en el entorno de un sistema de valoración integrada". Trabajos de Prehistoria 62 (2): 7-23. https://doi.org/10.3989/tp.2005.v62.i2.66

Ruiz Zapatero, G. 2016: "Publicar revistas de arqueología: cartografía académica y retos de futuro". Revista d'Arqueologia de Ponent 26: 265-279. https://doi.org/10.21001/rap.2016.26.15

Sanz Menéndez, L. 2019: "Reconocer al genio antes de que sea viejo", El Pais, 28 de febrero. https://elpais.com/sociedad/2019/02/27/ actualidad/1551287632_338104.html

Vidal Liy, J. I. y Rodríguez Yunta, L. 2015: Categorización de las revistas españolas de Arqueología y Prehistoria: Propuesta de indicadores sobre trayectoria, apertura de la autoría e internacionalidad en el periodo 2004-2013. Madrid, CCHS-CSIC, Documento de trabajo ISOC 2015/04. Disponible en http://hdl.handle.net/10261/111062

Trab. Prehist., 76, N. ${ }^{\circ}$ 2, julio-diciembre 2019, pp. 199-218, ISSN: 0082-5638

https://doi.org/10.3989/tp.2019.12233 